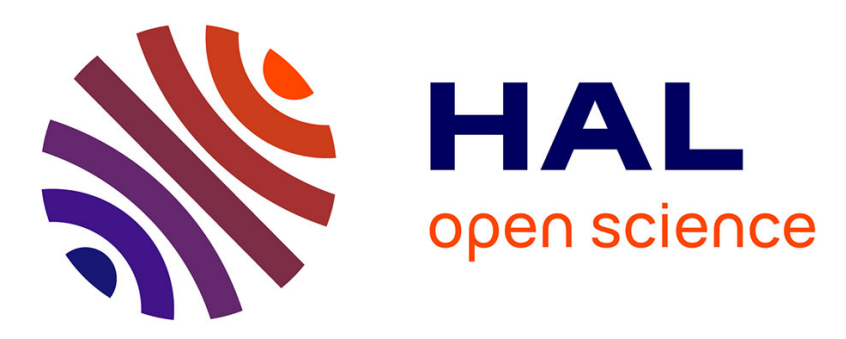

\title{
Self-consistent modeling of a surface AC dielectric barrier discharge actuator: In-depth analysis of positive and negative phases
}

Konstantinos Kourtzanidis, Guillaume Dufour, François Rogier

\section{To cite this version:}

Konstantinos Kourtzanidis, Guillaume Dufour, François Rogier. Self-consistent modeling of a surface AC dielectric barrier discharge actuator: In-depth analysis of positive and negative phases. Journal of Physics D: Applied Physics, 2021, 54 (4), pp.045203. 10.1088/1361-6463/abbcfd . hal-03131737

\author{
HAL Id: hal-03131737 \\ https://hal.science/hal-03131737
}

Submitted on 12 Mar 2021

HAL is a multi-disciplinary open access archive for the deposit and dissemination of scientific research documents, whether they are published or not. The documents may come from teaching and research institutions in France or abroad, or from public or private research centers.
L'archive ouverte pluridisciplinaire HAL, est destinée au dépôt et à la diffusion de documents scientifiques de niveau recherche, publiés ou non, émanant des établissements d'enseignement et de recherche français ou étrangers, des laboratoires publics ou privés. 


\title{
Self-consistent modeling of a surface AC dielectric barrier discharge actuator: In-depth analysis of positive and negative phases
}

\author{
K Kourtzanidis $\ddagger$, G Dufour, F Rogier \\ ONERA - The French Aerospace Lab, 31000, Toulouse, France \\ E-mail: kourtzanidis@certh.gr
}

\begin{abstract}
We report on a detailed numerical study of the two-phase operation of a surface Alternating Current (AC) Dielectric Barrier Discharge (DBD) actuator. We showcase that when the quasi-periodic regime has been established, residual volume and surface charges play an important role on the discharge evolution strongly coupling the positive and negative phases. It is shown that the quasi-neutral streamer discharge found on the positive phase serves as both a positive and negative charge generator and acts as a virtual anode. As the streamer is not attached to the dielectric surface, most of the surface charging occurs during its after-burn (relaxation) phase. The positive surface charge leads to an distant zone of high electric field and thus ion drift but also interacts majorly with the negative discharge phase. During the latter, microdischarges form near the active electrode and an intense cathode layer feeds with charges the discharge volume. Each microdischarge is followed by a plasma layer formation attached to the dielectric layer expanding further at each repetition until it occupies a volume linked to the streamer elongation length and positively charged surface portion. The strong coupling between the positive and negative phases along with the strong impact of the streamer discharge on both suggest implications that have been ignored so far in terms of EHD force production and its spatiotemporal distribution.
\end{abstract}

Submitted to: J. Phys. D: Appl. Phys.

$\ddagger$ Present address: CERTH - Centre for Research and Technology Hellas, 57001 Thermi, Thessaloniki, Greece 


\section{Introduction}

Plasma based actuators for flow control have been studied both experimentally and numerically in the last decades. These actuators include microwave $[1,2,3]$, corona and DBD discharges as well as plasma synthetic jets (or sparkjets) $[4,5,3]$. Concerning subsonic conditions, corona and DBD discharges are the most prominent for flow control applications [6], [7]. Such discharges, when formed on a surface mode, can transfer momentum to the surrounding gas (usually atmospheric pressure air) resulting to steady or pulsating wall-jets. As the jet formation takes place inside a thin layer close to the external flow boundary layer, various aerodynamic flow control mechanisms can be triggered. Such mechanisms include flow reattachment, control of laminar to turbulent transition, vortex generation etc. The development of a surface AC-DBD actuator by Roth et al. [8] has initiated a series of research projects due to a variety of advantages over corona based actuators. Surface AC-DBD actuators are almost non-intrusive to the flow and they can operate under high voltages preventing arc transition (and consequent dielectric breakdown). This operation leads to higher induced jet flows ("ionic wind") and thus higher control authority. Typical applied voltages for AC-DBD actuators are in the range of $5-25 \mathrm{kV}$ with $\mathrm{AC}$ frequencies of 1-25 kHz. Typical maximum induced velocities are in the range of $3-8 \mathrm{~m} / \mathrm{s}$.

The general physics behind the induced flow is quite well understood nowadays. The positive and negative ions produced during the discharge half phases accelerate due to the Coulomb force and transfer momentum to neutral particles via collisions. Nevertheless, the complexity of the discharge formation and consequent kinetic energy transfer in both positive and negative going cycles is yet to be fully revealed. Indeed, experiments have demonstrated that the plasma discharge nature is very different in both half-phases [9]. In the positive going cycle, high current streamer discharges form above the dielectric layer while in the negative going cycle, lower current but higher frequency microdischarges are present. Both regimes are strongly influenced by the presence of the dielectric materials and consequent surface charging cycles. Moreover, the periodic nature of the AC-DBD operation leads to strong interference between each cycle due to memory effects especially under high frequency actuation. The electrohydrodynamic (EHD) force produced is directly linked to this complex interactions and sub-cycle discharge behaviour leading to unsteady flow generation as demonstrated experimentally [10]. Consequently, the detailed study of each sub-cycle in terms of discharge evolution, charge and electric field re-distribution is essential towards a comprehensive operational analysis and optimization. While experiments can reveal important aspects of such operation they tend to focus on macroscopic and averaged attributes. On the other hand, numerical modeling can penetrate into refined details and provide additional insights not only on the sub-cycle discharge nature but also on the temporal and spatial distribution of the EHD force and resulting jet flow.

A first attempt to a very simplified modeling of the AC DBD actuator was per- 
formed in Ref. [11] but the strong assumptions made in the model cannot describe properly the full cycle operation. To tackle this problem, Boeuf et al. [12, 13] have performed self-consistent simulations of several AC cycles and retrieved the temporal distribution of EHD force as well as the two regimes of the plasma discharge. Despite the pioneering work, these simulations suffer from low accuracy mostly due to the numerical schemes used and concrete information on the two-phase operation is hard to extract. Similar simulations have been performed from several groups (Ref. [14, 15, 16]) but once again important insights are missing. Soloviev et al. have also performed very interesting modeling attempts [17] leading to simplified analytical models [18] for thrust production without a clear connection of the two sub-cycle regimes. Nishida et al. $[19,20]$ have also extensively studied the DBD actuator through two and three dimensional simulations, contributing towards the understanding of important aspects of its operation (three-dimensional effects, operational modes, frequency dependence). Despite all these efforts, the exact details of the two-phase operation especially under high voltage and high frequency remain ambiguous. The numerical and physical modeling challenges of AC DBD simulations are quite strong and thus advanced models need to be developed in order to confidently extract insights.

To this respect, we present here a detailed self-consistent numerical study of the AC surface DBD actuator. Taking advantage of advanced numerical techniques and refined spatiotemporal discretization the full $\mathrm{AC}$ cycle is modeled in detail revealing the importance and interconnection of both sub-cycles into the discharge evolution. The effects of the streamer and microdischarge regimes along with surface charging is underlined. The article is structured as follows: In section 2 we present briefly the physical and numerical models used. In section 3, we present the simulation case. In section 4 we firstly present the plasma characteristics during an AC cycle (subsection 4.1) and then focus on the positive phase - streamer(subsection 4.1.1) and negative phase - microdischarge regime 4.1.2). A discussion on limitations and assumptions made is presented in 5 . In section 6 we conclude and propose future directions.

\section{The COPAIER plasma solver}

The computational solver used in this paper is COPAIER [21], a multi-species and multitemperature plasma fluid solver allowing for self-consistent description of the plasma spatial and temporal evolution. COPAIER main features are summarized in Table 1.

In general, COPAIER is a robust plasma-fluid modeling tool integrating advanced numerical and physical modeling know-how. In the next subsections, the physical and numerical models used in this work are described in more detail. The equations provided below describing the models pertain to the two-dimensional (2D) formulation. 
Table 1. Main features of the COPAIER solver.

\begin{tabular}{ll}
\hline Formulation & 2D and 2.5D \\
\hline Mesh support & Structured/Unstructured/Hybrid \\
\hline Accuracy & 2nd order (in space and time) \\
\hline LFA/LMEA & Both (user choice) \\
\hline Photoionization & Yes (user choice) \\
\hline \# species and reactions & Arbitrary (tabulated - user choice) \\
\hline Parallel & Yes (MPI) \\
\hline
\end{tabular}

\subsection{Physical model}

The fluid (continuum) description of the plasma is the core of the physical model used in COPAIER. Each of the species, $s$, considered in the pre-defined plasma-gas chemistry is governed by a continuity equation :

$$
\frac{\partial n_{s}}{\partial t}+\vec{\nabla} \cdot \vec{\Gamma}_{s}=S_{s}
$$

where $S_{s}$ is the net rate of production of species $s$ due to chemical reactions and it reads :

$$
S_{s}=\sum_{r} c_{s, r} R_{r}+S_{p h}
$$

where $c_{s, r}$ is the net number of particles of species $s$ created or lost in one reaction of type $r$ (so it can be positive or negative). Note that $S_{p h}$ is the photo-ionization source term based on the three-group first-order Eddington approximation model [22] (details are omitted here as photo-ionization is not included in this study). The user has two possibilities concerning the reaction rate $R_{r}$ : It is either proportional to the densities of the reacting species, which for a two-body reaction reads $R=k n_{1} n_{2}$ where $k$ is the reaction rate coefficient in units of $m^{3} / s$ or written as $R=\alpha|\vec{\Gamma}| n_{2}$ in the case where the Townsend coefficient $\alpha$ (in units of $m^{2}$ ) is known (with the total flux corresponding to electron impact reactions). We note that the use of Townsend coefficients can increase the stability of the numerical scheme when the electron flux is field driven as in the case of DC and low frequency AC discharges and thus we have chosen Townsend coefficients for the ionization processes (See below).

The species number flux $\vec{\Gamma}=n_{s} \overrightarrow{v_{s}}$ is given by the momentum balance equation which is approximated by the drift-diffusion equation :

$$
\overrightarrow{\Gamma_{s}}=\operatorname{sgn}\left(q_{s}\right) n_{s} \mu_{s} \vec{E}-\vec{\nabla}\left(n_{s} D_{s}\right)-n_{s} \vec{u}
$$

where $q$ is the species charge, $\mu_{s}$ and $D_{s}$ are the species $k$ mobility and diffusion coefficient respectively, $u$ is the mean mass fluid convection velocity. The electric field is 
defined as $\vec{E}=-\nabla \Phi$, with $\Phi$ being the electrostatic potential defined by the Poisson's equation

$$
\nabla \cdot(\epsilon \nabla \Phi)=-\rho=-\sum_{s} q_{s} n_{s}
$$

where $\epsilon$ is the dielectric permittivity.

In Eq. 3 the diffusion coefficient is related to the mobility term by Einstein's relation, $D_{s}=\mu_{s} k_{B} T_{s} / q$. The rest of transport (mobility $\mu$ ) and reaction rate coefficients $(k)$ are tabulated with an electric energy dependence under the Local Mean Energy Approximation (LMEA - the Local Field Approximation is also available).

The Local Field Approximation (LFA) is often not valid for electrons due to the poor energy transfer in electron-neutral collisions owing to the huge mass difference. The electron temperature can not be approximated using the LFA which fails in regions where nonlocal electron energy transport effects are dominant (high electric field regions) [23]. In such cases, transport and rate coefficients depend on the mean electron energy instead of the electric field. Under this Local Mean Energy Approximation (LMEA) an energy balance equation needs to be solved for the electrons.

Here, the mean electron temperature can be obtained by the electron energy conservation given as :

$$
\frac{\partial n_{\epsilon}}{\partial t}+\vec{\nabla} \cdot \vec{\Gamma}_{\epsilon}=S_{\epsilon}
$$

where $n_{\epsilon}=n_{e} \epsilon$ is the electron energy density and $\epsilon$ is the electron mean energy which by assuming that is a results mainly from random motion relates to the electron mean temperature by $k_{B} T_{e}=2 / 3 \epsilon$.

The electron energy flux reads :

$$
\vec{\Gamma}_{\epsilon}=\frac{5}{3} \epsilon \vec{\Gamma}_{e}+\vec{q}
$$

The heat flux, $\vec{q}$ is assumed proportional to the gradient of the electron mean energy :

$$
\vec{q}=-\frac{5}{3} n_{e} D_{e} \nabla \epsilon
$$

The source term, $S_{\epsilon}$ includes heating by the electric field (electron Joule heating) and energy losses in inelastic and elastic collisions as:

$$
S_{\epsilon}=-e \vec{\Gamma}_{e} \cdot \vec{E}-n_{e} \sum_{s} \epsilon_{s} k_{s} n_{s}-\frac{3}{2} k_{b} n_{e} \frac{2 m_{e}}{m_{s}}\left(T_{e}-T_{g}\right) \nu_{m}
$$

where $\nu_{m}$ is the electron momentum-transfer frequency. The last term, corresponds to the energy loss due to elastic collisions which can also be approximated with an effective rate coefficient and integrated directly to the inelastic energy loss term. The LMEA has been used in this work.

The density of the background species is given by the ideal gas law :

$$
p=\sum n_{s} k_{B} T_{s}
$$

In this work, air under atmospheric pressure (760 torr) is represented by a $79 \% \mathrm{~N}_{2}$ and $21 \% \mathrm{O}_{2}$ gas mixture. 


\subsection{Boundary conditions}

Boundary conditions for the transport equations of each species are imposed on the species flux. Depending on the boundary we distinguish 3 BCs :

- Free flow, $\nabla n_{s} \cdot n=0$

- Solid surface (electrons)

$$
\vec{\Gamma}_{e} \cdot \vec{n}=\frac{1}{2} v_{t h} n_{e}-\alpha \sum_{s} \gamma_{s} \vec{\Gamma}_{s} \cdot \vec{n}
$$

- Solid surface (rest)

$$
\vec{\Gamma}_{s} \cdot \vec{n}=\frac{1}{2} v_{t h} n_{s}+\alpha \mu_{s}(\vec{E} \cdot \vec{n}) n_{s}
$$

We assume that each particle is instantaneous recombined on a solid surface (or stored in the case of dielectrics, see below). Its flux is deduced from kinetic considerations under relevant approximations (based on Ref. [24]). We start by writing the net flux at the wall as :

$$
n v_{s} \cdot \vec{n}=\mu_{s} n_{s} \vec{E}-D_{s} \vec{\nabla} n_{s}=n_{s} v_{e f f}
$$

where $v_{\text {eff }}$ is an effective velocity for the incident particles, $v_{s}$ is the mean (driftdiffusion) velocity of each particle $s$, and $\vec{n}$ is the normal vector pointing towards the wall.

For electrons, we assume a Maxwellian distribution function including directed motion (drift from the electric field) and after a two-term development (assuming collisional electrons) we obtain the effective incident velocity which reads :

$$
v_{e f f}=\frac{1}{4} v_{t h}+\frac{1}{2} \overrightarrow{v_{e}} \cdot \vec{n}=\frac{1}{4} v_{t h} \pm \frac{1}{2} \mu_{s} \vec{E} \cdot \vec{n}-D_{e} \frac{\vec{\nabla} n_{e}}{n_{e}} \cdot \vec{n}
$$

From Eq. 12 and 13, we obtain the flux expression in Eq. 10. For the rest particles, the two-term approximation fails and thus we are using a combination of drift and thermal motion flux (Eq. 11).

The thermal velocity is taken as $v_{t h}=\sqrt{\frac{8 k_{B} T_{s}}{\pi m}}$. The coefficient $\alpha$ takes the value 1 if the drift velocity is directed towards to the solid surface and its 0 otherwise.

Moreover, for electrons, the solid surface flux is modified to include the flux due to secondary electron emission : the average number of electrons emitted per incident ion is given by the secondary emission coefficient $\gamma_{s}$.

The following relation is used for the ion temperature [25]:

$$
k_{B} T_{s}=k_{B} T_{g}+\frac{m_{s}+m_{g}}{5 m_{s}+3 m_{g}} m_{g}\left(\mu_{s} E\right)^{2}
$$

where $T_{g}$ and $m_{g}$ denote the gas temperature and mass respectively.

Surface charge accumulation on dielectric surfaces is taken into account by assuming that particles stick to the dielectric surface and no diffusion occurs. Thus the surface charge is given by:

$$
\sigma=\int \vec{j} \cdot \vec{n} d t
$$


where the plasma current density reads :

$$
\vec{j}=\sum_{s} q_{s} \vec{\Gamma}_{s}
$$

\subsection{Numerical models}

A detailed description of the numerical schemes used in the COPAIER solver can be found in Ref. [21]. Here we summarize the main features along with recent advances. A splitting technique is used for the coupling between drift,diffusion, kinetics and the Poisson equation. The Strang splitting strategy, which guarantees a second-order accurate coupling, has been used. A finite volume approach is used to solve the driftdiffusion equations. Second-order accuracy both in space and time is ensured via MUSCL reconstruction for the convective fluxes, a RKL formulation with super timestepping [26] for the diffusive fluxes and an explicit time-integration based on Heun's method. A sub-cycling time-integration strategy has been adopted, considering the large discrepancy between electron and heavy species characteristic time-scales. In Eq. 5 and 8 , the evaluation of the energy loss source term explicitly can lead to non-physical oscillations that can damage the stability of the whole system. In order to circumvent this issue, an implicit treatment of the inelastic energy loss and joule heating source terms has been adopted based on Ref.[27]. The Finite Element Method has been used to solve the Poisson equation. In order to remain consistent with the accuracy provided by the numerical scheme used for the transport of the species, a P1-Finite Element formulation has been implemented for the unstructured mesh, while a Q1 formulation has been used for the structured part of the mesh. In order to limit the computational burden imposed by this method when refined meshes are used, a non-overlapping domain decomposition method has been developed. Depending on the size of the mesh, either a direct LU decomposition or an iterative Conjugate Gradient Method can be used. Finally, considering the kinetics dynamics, a second-order explicit Runge-Kutta method has been developed. This method is based on the estimation of a maximum time step ensuring the positivity of the components of the kinetics.

We note here that the choice of an explicit time-integration might seem illogical in plasma-fluid simulations. Indeed, the CFL stability criteria impose severe limitations on the time-steps (especially on the electron and electron energy transport), rendering the CPU burden quite important. Several authors have used implicit or semi-implicit formulations to overcome the CFL stability issues. Nevertheless, such implicit based schemes suffer from important numerical diffusion errors especially under conditions where the relevant physics are strongly coupled (streamer propagation for example). In this work, we are interested in a detailed description of the plasma generation (and consequent EHD force production see Ref. [28]) and thus we choose to use numerical schemes that can provide the accuracy needed. Moreover, several authors have used a semi-implicit formulation of Poisson's equation under an asymptotic preserving approach $[29,30]$. Such approach, relaxes the stability criterion on the dielectric 
relaxation time [31] :

$$
d t_{d} \leq \frac{\epsilon_{0}}{\sum_{s}\left|q_{s}\right| \mu_{s} n_{s}}
$$

The dielectric relaxation time (Eq. 17) is a physical time. Therefore, even if the semiimplicit method relaxes the stability criterion allowing for larger time-steps, the physical quantities of interest are very ofter linked to the physical time of the problem. Attention needs to be paid when using the semi-implicit formulation in respect to the accuracy of the obtained results. Therefore in this work, even if the semi-implicit approach is available in the COPAIER framework, an explicit formulation has also been used for solving Poisson's equation.

The combination of advanced physical and numerical models render our simulations quite robust and accurate, being able to capture the complex evolution of the surface AC-DBD.

\section{The DBD under study, computational and initial parameters}

The AC-DBD actuator consists of two planar electrodes separated by a dielectric layer. The exposed electrode is subject to the AC-High Voltage (HV) source while the embedded one is grounded. A 2D-view of the AC-DBD is shown in Fig. 1. The operation of the AC-DBD results to a wall jet flow parallel to the dielectric surface.

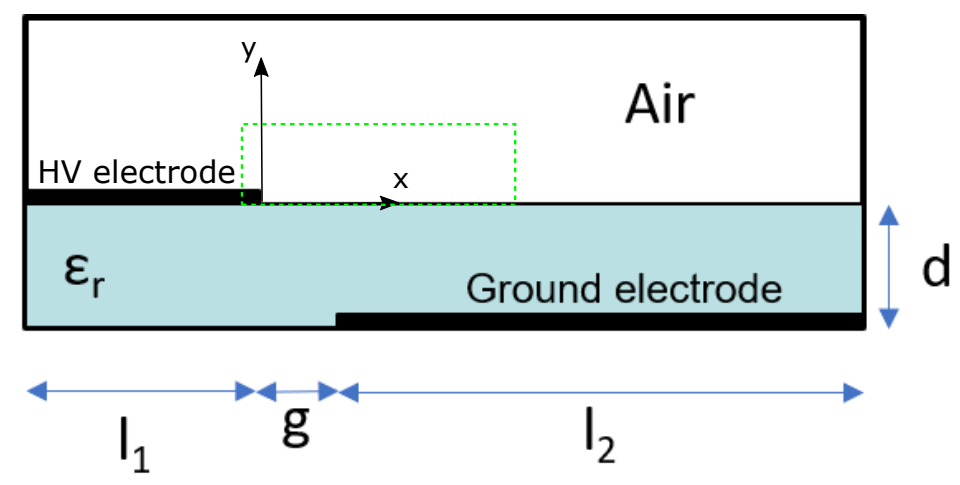

Figure 1. The AC-DBD actuator and full simulation domain along with dimensions as stated in Table 2. The dashed rectangle depicts the zoomed region shown in the Numerical Results section figures (Sec. 4) for visualization purposes (not in scale).

In the present study, we focus on a geometrical configuration similar to the ACDBD used in Ref. [32] and the ones described in Ref. [9]. The AC frequency used in the simulations is increased compared to the experimental results of Ref. [32] (100 kHz instead of $1 \mathrm{kHz}$ ) in order to reduce the CPU time and obtain qualitative results. These results offer important insights on the physics of both positive and negative going phases while the AC frequency should only affect quantitatively the obtained results. Moreover 
Table 2. Geometrical and operational conditions for the simulations.

\begin{tabular}{ll}
\hline Frequency & $100 \mathrm{kHz}$ \\
\hline Voltage (max) & $20 \mathrm{kV}$ \\
\hline Electrode thickness & $70 \mu \mathrm{m}$ \\
\hline HV electrode length & $l_{1}=5 \mathrm{~mm}$ \\
\hline Ground electrode length & $l_{2}=1 \mathrm{~cm}$ \\
\hline Inter-electrode gap & $g=1 \mathrm{~mm}$ \\
\hline Relative permitivity of dielectric & 3.2 \\
\hline Dielectric thickness & $d=3 \mathrm{~mm}$ \\
\hline Electric circuit resistance & $100 \Omega$ \\
\hline
\end{tabular}

the maximum applied voltage is reduced to $15 \mathrm{kV}$ instead of $20 \mathrm{kV}$ in order to retain the physical regime which is experimentally observed (the potential slope DV/Dt is one of the critical parameters for the number of streamer and microdischarge repetition as well as memory effects but the nature of the discharge regimes should not change as long as sufficient time for dielectric discharging is present). A more detailed discussion on the effect of $\mathrm{AC}$ frequency in the results compared to experimental studies in lower frequencies (1-25 kHz) and all assumptions made in the model is presented in Section 5 .

In Table 2, we summarize the geometrical and operational conditions for the simulations (see also Fig. 1).

The computational mesh is a structured, non-uniform one created in the Gmsh [33] software, properly refined near the exposed electrode edge and near the dielectric surface. It consists of 150.000 cells. The minimum cell size is approx. $1 \mu \mathrm{m}$ at the 'boundary layer' zone above the dielectric surface in order to resolve all plasma-related aspects such as streamer sheaths and cathode layer formation. The use of such refined computational mesh is of utmost importance and can eliminate large errors not only on quantitative analysis but also on a qualitative level - the initiation of streamers and microdischarges, streamer elongation, sheath formulation and dielectric charging strongly depend on this level of refinement. The domain has been decomposed in 96 sub-domains and the calculations were run on ONERA's SPIRO cluster over 96 procs.

We choose to use a simplified air-plasma chemistry following the work of Boeuf et al. $[12,13]$. Without loss of generality, such chemistry allows for a proper description of the main charged species production, discharge evolution and qualitative EHD force production while largely reducing the CPU burden of the simulation. Transport and reaction rate coefficients have been extracted using BOLSIG $+[34,35,36]$ and properly tabulated into a COPAIER compatible format. The plasma related conditions and chemistry used in the simulations are summarized in Table 3 (SEE stands for the Secondary Electron Emission coefficient). A discussion on the choice of chemistry is given in Sec. 5 . 
Table 3. Chemistry and plasma related parametres used in the simulations.

\begin{tabular}{lc}
\hline Species & $\begin{array}{c}\text { electrons }(\mathrm{e}), \text { negative ions }\left(n_{i}\right), \\
\text { positive ions }\left(p_{i}\right) \text { and neutrals }\end{array}$ \\
\hline Reactions & $\begin{array}{c}\text { ionization, 2-body attachment, } \\
\text { 3-body attachment, } \\
\text { e-ion recombination, } \\
\text { ion-ion recombination }\end{array}$ \\
\hline Floor density for e and $n_{i}$ & $5 \times 10^{11} \mathrm{~m}^{-3}$ \\
\hline Floor density for $p_{i}$ & $10^{12} \mathrm{~m}^{-3}$ \\
\hline SEE coef. for electrode & 0.05 \\
\hline SEE coef. for dielectric & 0.01 \\
\hline
\end{tabular}

\section{Numerical Results}

\subsection{Electrical properties and discharge evolution}

Fig. 2 shows the conduction current versus the simulation time for the first 3.5 periods (the voltage waveform is also plotted with the dashed line for visualization purposes). After the initial transient regime corresponding to the 1st period, a semi-periodic regime is established. Two distinct discharge regimes are captured : In the positive phase, a single high current streamer discharge is formed while a series of microdischarges are present in the negative phase. The conduction current pattern is repeated in each period of actuation. The streamer current reaches approximately $9 \mathrm{~A} / \mathrm{m}$ while the microdischarges carry currents in the order of $0.5-2.5 \mathrm{~A} / \mathrm{m}$ (here the current is given in units of $\mathrm{A} / \mathrm{m}$ due to the $2 \mathrm{D}$ approximation ). Taking into account the $2 \mathrm{D}$ approximation used, we can try to extrapolate the total current of the 3D configuration based on the findings of Ref. [9] (see Fig. 4 of this reference). The total current can be found by multiplying the calculated one with the z-extension of the actuators which is $0.12 \mathrm{~m}$. This total current then ranges between 60 and $1000 \mathrm{~mA}$. Noting that in reality the plasma discharge takes the form of filaments and/or spotted discharges instead of a uniform plasma sheet and assuming that there are approximately 10 discharges per actuator span we can assume that the total current ranges between 6-100 mA which is is good agreement with the measured currents. Of course such approach is quite simplified but it provides validation to our findings to some extent.

To avoid ambiguities, we clarify here that positive phase denotes the time-window when the conduction current is positive and respectively for the negative phase. This is a clear distinction of the two phases as any other categorization (voltage slope sign, voltage sign etc) is not easily comparable and can lead to interpretation problems. As the dielectric surface is being periodically charged either positively or negatively and due to the potential modification and memory effects associated to this charging, the sign change of the conduction current does not follow the voltage or its slope sign. These 
quasi-periodic features, allow us to focus on one period of actuation and investigate in detail the discharge behaviour. For the rest of the paper, we choose the 3rd period as the quasi-periodic regime has already been estabilshed. Fig. 3, shows the conduction current during the 3rd period of the AC actuation. Once again the single streamer during the positive phase and four microdischarges of reduced current during the negative phase are clearly visible. The figure is characteristic of a typical high-voltage surface DBD actuator current during one period [9]. Evidence of the great importance of the dielectric charging and its memory effects can be easily identified by studying the potential distribution at the beginning of the positive and negative phase. Fig. 4 shows the potential distribution along with EHD force vectors while Fig. 5 the surface charge on the dielectric surface at time-instants corresponding to the beginning of the two phases as defined above. The instantaneous EHD force vector per unit volume is given by:

$$
\vec{F}_{E H D}=\rho \vec{E}
$$

where $\rho$ is the total charge density. The EHD force vectors provide an indication of the charge drift direction and magnitude at each instant as they link the regions of high electric field and high charge population. We note that the potential distribution is strongly modified by the residual dielectric charge at both phases. At the beginning of the positive phase, a substantial portion of the dielectric layer is negatively charged (length of approx. $2 \mathrm{~mm}$ ). The potential produced by the surface charge is higher than the negative potential applied on the HV electrode leading to the initiation of the positive phase. At this time-instant, electron population is very low and only positive and negative ions contribute to the EHD force. At the near electrode region positive ions drift towards the active electrode and the negatively charged dielectric portion while negative ions are repelled by the latter at a distance of several millimeters ending their trajectory on the dielectric surface downstream the negatively charged region.

At the beginning of the negative phase, the process is more or less reversed. The surface charge is now positive capturing a length of more than $3 \mathrm{~mm}$. As electron population is still very low, it is now negative ions that dominate in the regions near the active electrode and close to the dielectric surface - they drift towards the positively charged region of the dielectric. The surface charge (Fig. 5) at both time-instants showcase that dielectric charging is not uniform especially close to the active electrode (distance of less than $1 \mathrm{~mm}$ which corresponds to the gap length). This is due to the structural behavior of the discharge during each subcycle and thus a more detailed analysis is necessary to assess such observation.

The streamer and microdischarge regimes will prove essential in understanding the exact complex interaction between charge production, dielectric charging and electric field distribution. Therefore, in the following subsections, we focus separately on the positive and negative phases and examine in depth the discharge nature, focusing on the streamer (positive phase) discharge and one single microdischarge (negative phase). We note here that as we are considering the semi-periodic regime of the actuator, both phases are coupled and thus we urge the reader to consider the following subsections in a 


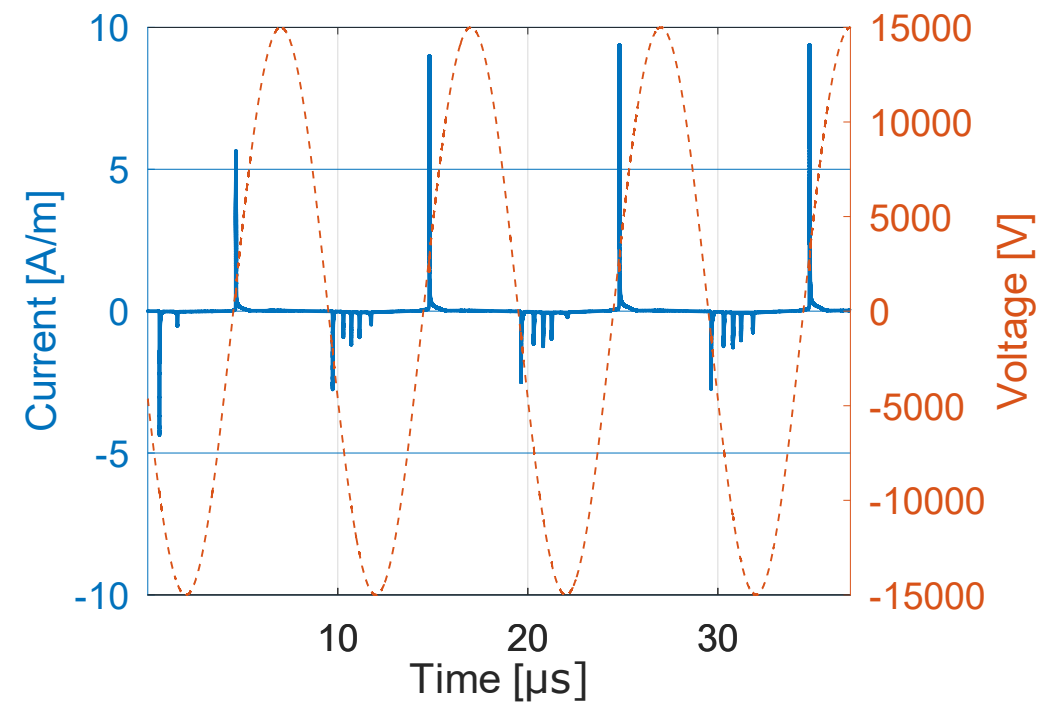

Figure 2. Current vs Time

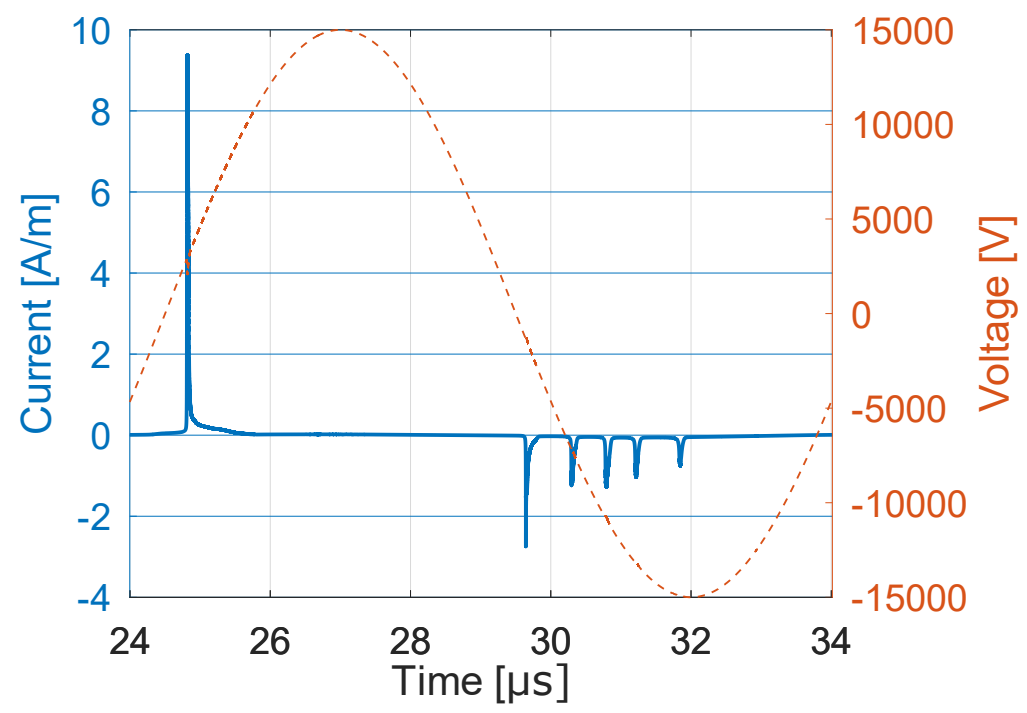

Figure 3. Current in 3rd period vs Time

holistic way. The reader can find a video of the full cycle electron temperature evolution as supplementary material where both phases and regimes are clearly distinguished.

4.1.1. Positive phase - Streamer discharge We emphasize again that positive phase denotes the time-window when the total conduction current is positive as shown in Fig. 6. Our focus will be targeted to the streamer pulse as shown in the embedded zoomed figure in Fig. 6. 


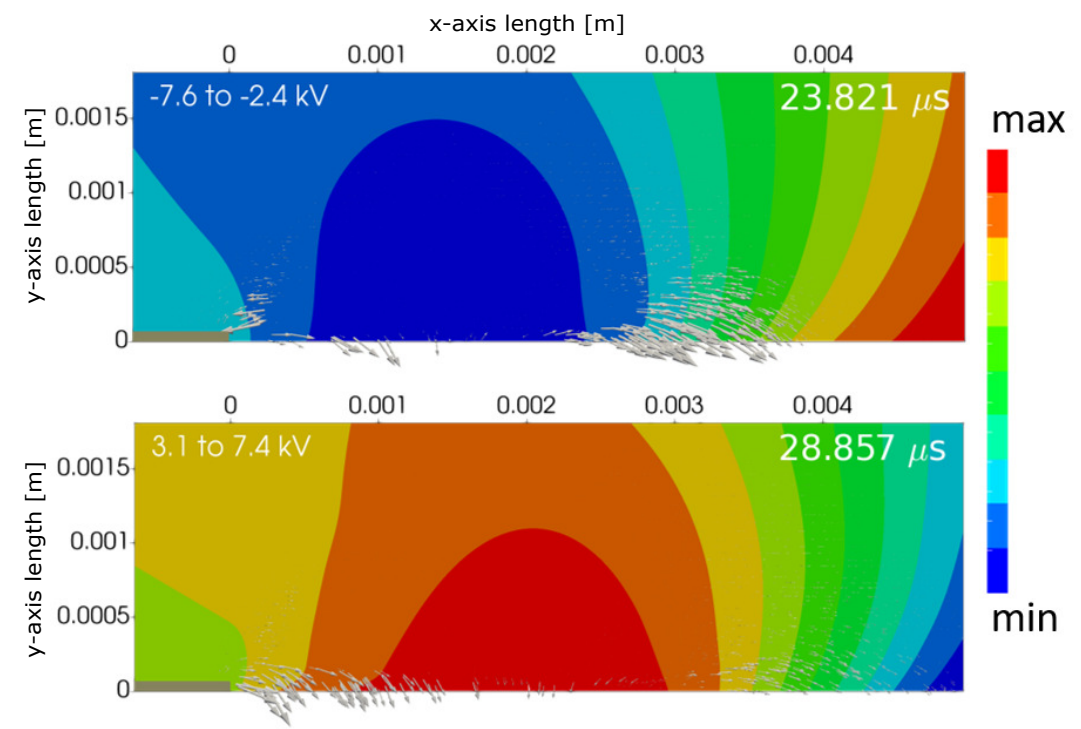

Figure 4. Potential distribution $[\mathrm{V}]$ (min and max values are indicated on the topleft corner of each time-snapshot) at the beginning of the positive $(t=23.821 \mu \mathrm{s})$ and negative $(t=28.857 \mu \mathrm{s})$ phase. The EHD force vector field is also superimposed (vectors are scaled by their magnitude: Min value $=0 \mathrm{~N} / \mathrm{m}^{3}$, Max values are $3.4 \times 10^{5}$, $2.2 \times 10^{5} \mathrm{~N} / \mathrm{m}^{3}$ from top to bottom). The dielectric surface charging results to the negative and positive potential zones shown respectively.

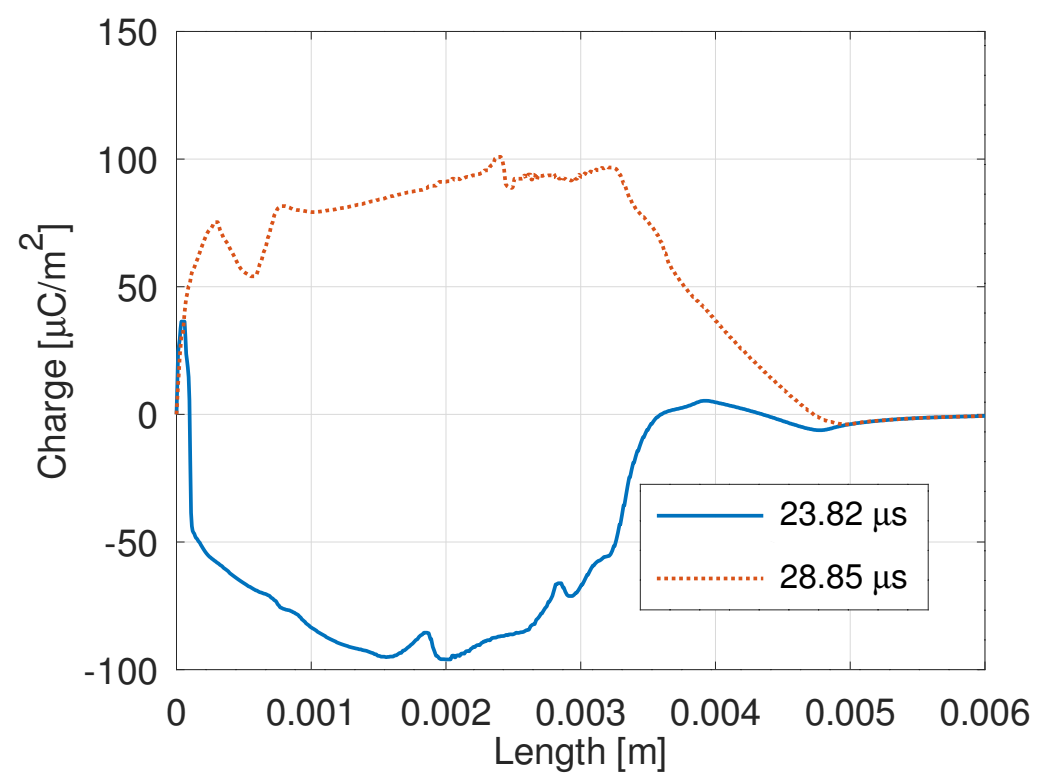

Figure 5. Surface charge at the beginning of the positive and the negative phases. The charging portion of the dielectric expands to more than $3 \mathrm{~mm}$.

At an early stage of this positive phase, the potential distribution is strongly modified by the charged portion of the dielectric layer as described in the previous subsection (see Fig. 4). Fig. 7 shows the electron temperature for different timeinstants during the early stage $(t=23.821 \mu \mathrm{s})$, the streamer formation $(t=24.809$ 


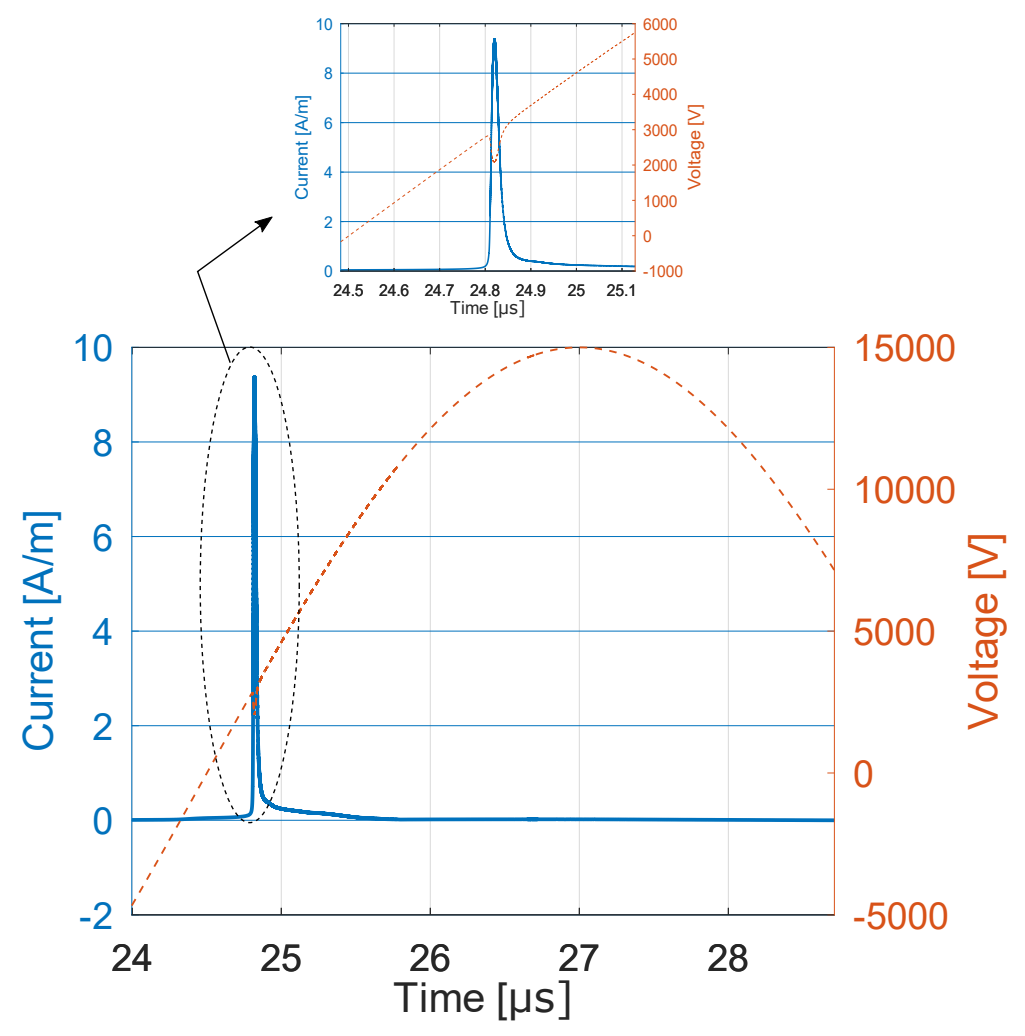

Figure 6. Current vs time in the positive phase. The zoomed embedded figure on the top shows the current vs time during the streamer discharge.

$\mu s)$, propagation $(t=23.821-24.850 \mu s)$ and relaxation $(t=25.552 \mu s)$. It is evident that at the early stage $(t=23.821 \mu \mathrm{s})$ there are two regions of high electron energy: one attached to the active electrode and one at a distance of approx. $3.5 \mathrm{~mm}$ from it. The latter is directly linked to the negatively charged region of the dielectric layer as seen in Fig. 5. As the applied potential increases, the electric field (and associated electron temperature) intensifies near the active electrode leading to increased local ionization. The discharge starts as a corona-like in nature. The ionization layer is attached to the exposed electrode edge. Electrons move fast towards the exposed electrode edge while positive ions drift towards the dielectric surface forming a curve path linked mainly to the $1 \mathrm{~mm}$ gap between both electrodes and the electric field lines due to the potential distribution from the negatively charged region (which persists from the negative phase) of the dielectric. Note that a portion of the positive ions formed in this ionization layer drift towards the dielectric, charging it mainly very close to the active electrode (at a distance of approx. $0.5 \mathrm{~mm}$, see Fig. 11). The curved path of the main positive ion drift leads to volumetric charge accumulation of positive charges at a distance of approx. 0.5 $\mathrm{mm}$ from the active electrode and a height of approx. $100 \mu \mathrm{m}((t=23.821 \mu \mathrm{s}))$. The volumetric space charge locally distorts the external electric field and leads to further local ionization. The evolution of the electron density from this time-instant $(t=24.809$ 


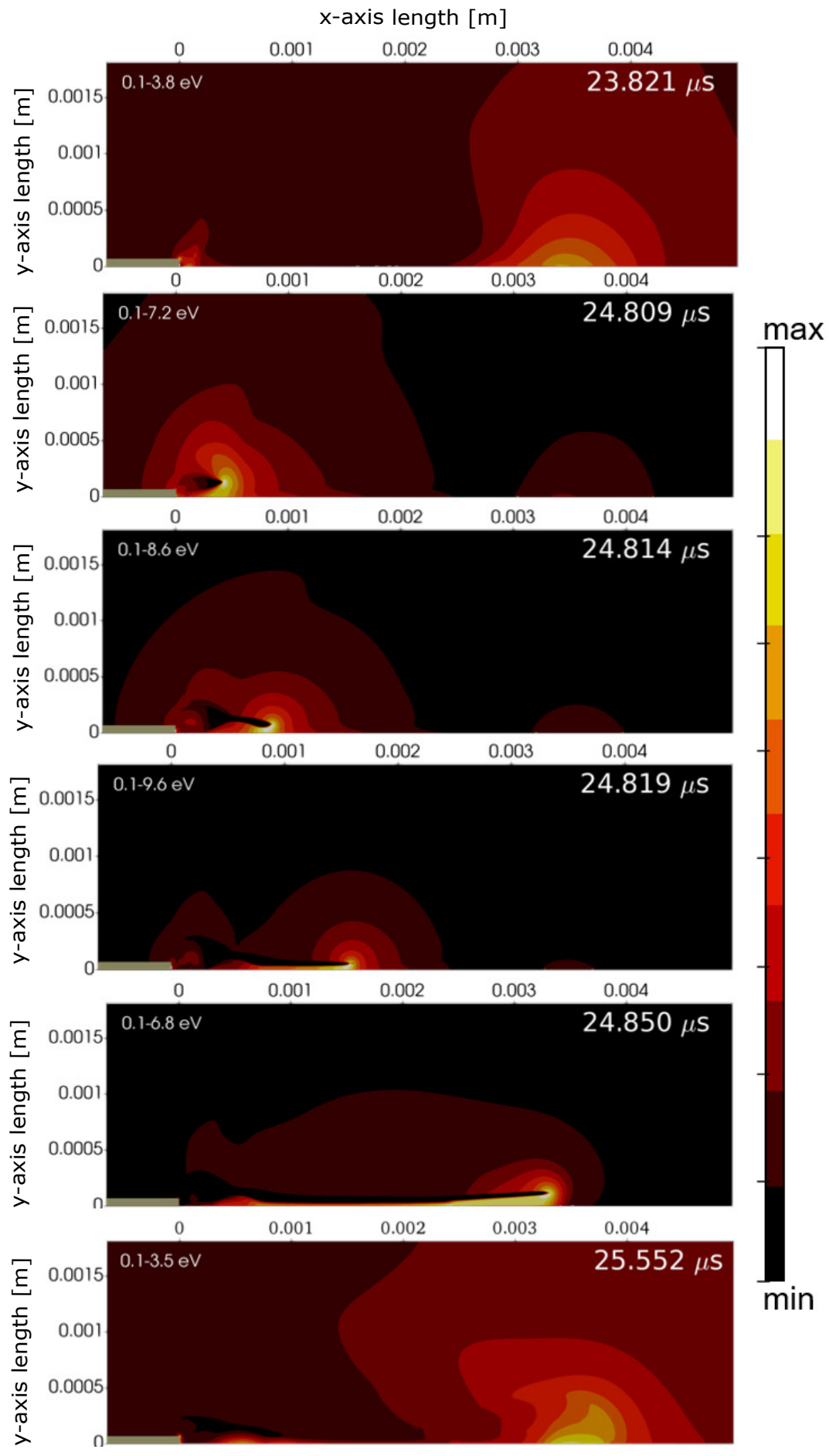

Figure 7. Electron Temperature $[\mathrm{eV}]$ for different times in the positive phase (streamer regime - min and max values are indicated on the top-left corner of each timesnapshot). The early stage $(t=23.821 \mu s)$ distribution of the electron temperature is also shown here. The development of the surface streamer discharge holding high electron temperatures around its head is apparent.

$\mu s)$ is shown in Fig. 8. When the space charge becomes sufficient, the positive going streamer is initiated. Initially, the streamer tends to approach the dielectric surface 


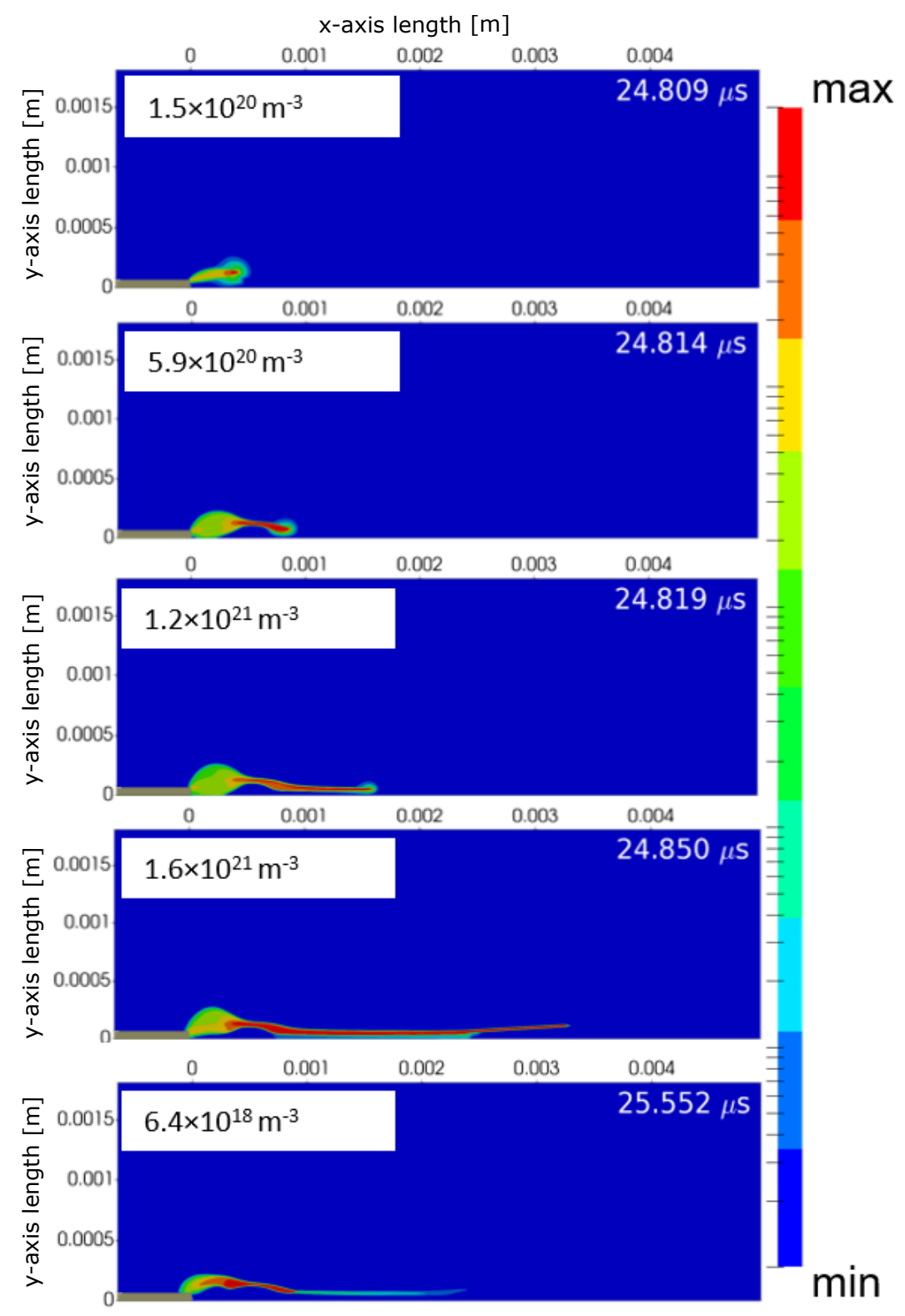

Figure 8. Electron density distribution $\left[\mathrm{m}^{-3}, \log\right.$-scale] for different times in the positive phase - streamer regime (scaled to minimum of $10^{14} \mathrm{~m}^{-3}$ for visualization purposes - max values are indicated on the top-left corner of each time-snapshot). The streamer discharge propagates over the dielectric surface detached until it reaches its maximum length of more than $3 \mathrm{~mm}$.

but it quickly changes direction and propagates parallel to the dielectric surface, fed by secondary electron emission from the dielectric. We note here that photoionization normally enhances the propagation but its broad effect can be also modeled with a high enough floor density for the electron species as done in this work (playing the role of seed electrons). The streamer is not attached to the dielectric but it propagates at a height of 10-20 $\mu \mathrm{m}$ which highlights the importance of a refined mesh near the dielectric surface. This non-attached propagation has important consequences on the surface charging. 
During its propagation, the streamer only slightly charges the dielectric surface mainly due to charge diffusion inside its body-sheath but it holds a positive potential due to potential diffusion from the active electrode and positive space charge around its body (sheaths). Fig. 9 shows the potential distribution along with EHD force vectors which support our claims.

The shielding of the electric field inside the streamer's quasineutral body prevents charge drift except from its head region where positive charge dominates but the fast propagation does not allow for efficient surface charging. The propagation is quite fast - the streamer has reached its full length of approx. $3.5 \mathrm{~mm}$ in less than $20 \mathrm{~ns}$ with an average velocity of $1.75 \times 10^{7} \mathrm{~cm} / \mathrm{s}$ close to velocities of cathode-directed streamers as observed in experiments [37]. Positive ions and electron densities reach values of $\approx 10^{21}$ $m^{-3}$ inside the streamer body, once again close to experimentally measured values for streamer propagation [37]. The electrons at the streamer head reach energies of more than $9 \mathrm{eV}$. Negative ions are also populating the streamer body with densities two orders of magnitude lower than the rest of charged species $\left(\approx 10^{18} \mathrm{~m}^{-3}\right)$. Higher negative ion population is found near the exposed electrode (inside the gap-distance) as negative ions drift towards this region and attachment dominates under the low electric field. The streamer propagation stops when the electric field at its head is not sufficient for further strong ionization. Nevertheless, the virtual anode formed by the streamer enhances the electric field at its head and inside its sheath (around the streamer body). Positive ions created or falling in these regions (due to ionization there and/or diffusion in the after-burn phase) are accelerated towards the dielectric surface ahead of the streamer final elongation length (Fig. $9-t=24.850 \mu s$ ).

The quasi-neutral plasma slowly decays due to recombination inside the streamer's body and diffusion until the electric field is not shielded anymore and electrons drift quickly towards the anode. This process lasts some 100s of ns, described here as the after-burn phase. It is during the after-burn phase that the dielectric layer is being strongly charged due to the diffusion and convection of positive charges remaining in the streamer's body. The positive charge occupies a volume linked to the streamer propagation length while negative charges populate mainly the gap region as shown in Fig. 10. Fig. 11 shows the surface charge on the dielectric surface right before and right after the streamer discharge formation. As the dielectric surface is being positively charged, a region of relatively high electric field (and relatively high electron temperature see Fig. 7) forms at the end of charged region. This charged region plays now the role of the virtual anode, leading to further ion convection at a distance of approx. $3.5 \mathrm{~mm}$ (evident from the EHD force vectors, Fig. $9-t=25.552 \mu s$ ). This fact implies strong consequences on the spatial distribution of the EHD force (see Ref. [28] for more details). As the discharge further relaxes and the applied potential continues to increase the process restarts with the corona-like discharge near the exposed electrode. The high actuation frequency does not allow for a second streamer discharge to be initiated due to the dielectric charging and consequent potential reversal towards the negative phase. 


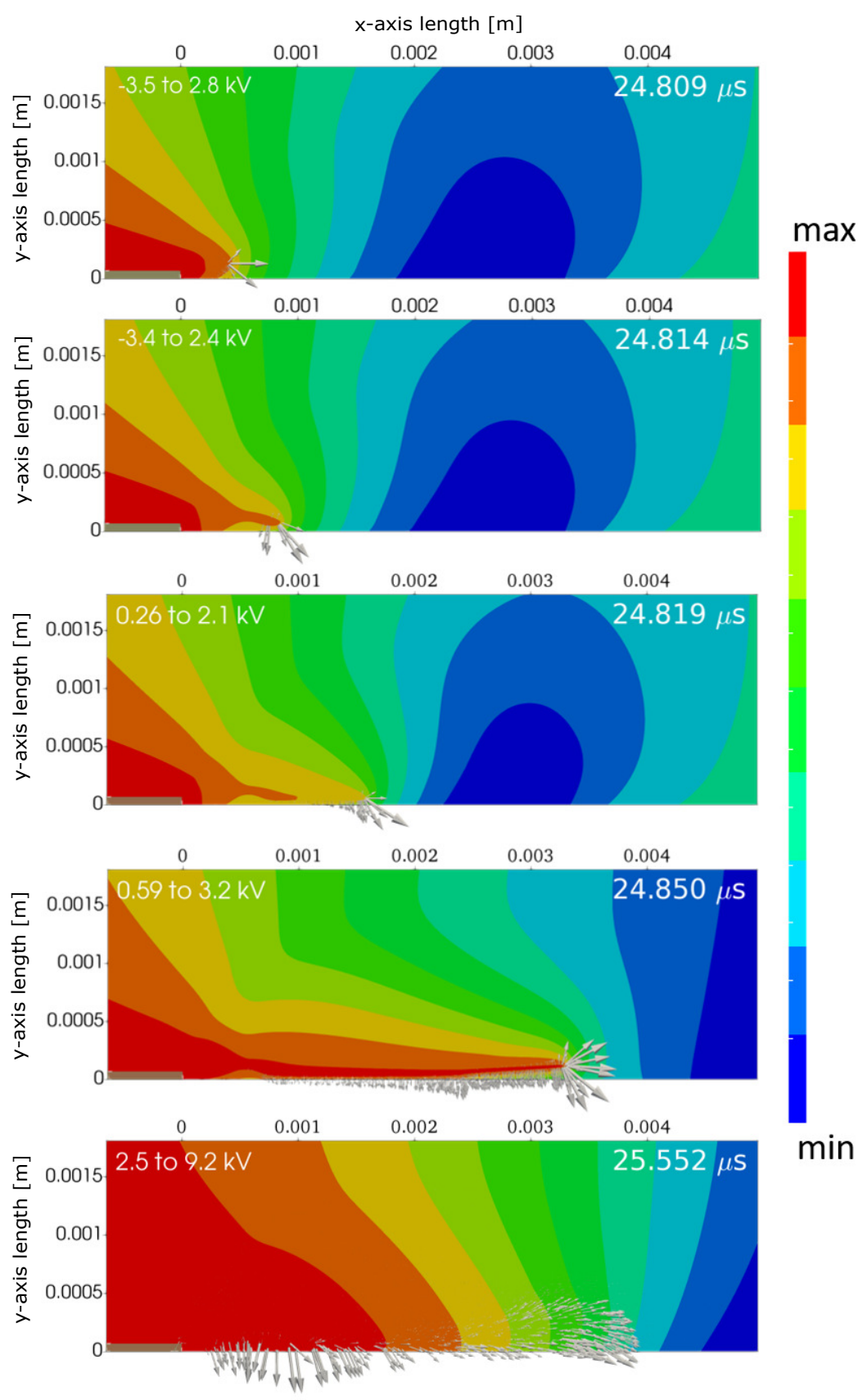

Figure 9. Potential $[\mathrm{V}]$ distribution for different times in the positive phase - streamer regime (min and max values are indicated on the top-left corner of each time-snapshot). The EHD force vector field is also superimposed (vectors are scaled by their magnitude: Min value $=0 \mathrm{~N} / \mathrm{m}^{3}$, Max values are $2 \times 10^{8}, 4.6 \times 10^{8}, 1 \times 10^{9}, 9.8 \times 10^{7}, 2.8 \times 10^{6} \mathrm{~N} / \mathrm{m}^{3}$ from top to bottom). The streamer holds a positive potential acting as a virtual anode during its propagation while it charges the dielectric surface during its relaxation.

4.1.2. Negative phase - Microdischarges The negative phase denotes the time-window when the total conduction current is negative (Fig. 12) and we now investigate in detail the physics of the negative microdischarge formation and without loss of generality, a single microdischarge is under focus. The repetition of such microdischarges is due to 


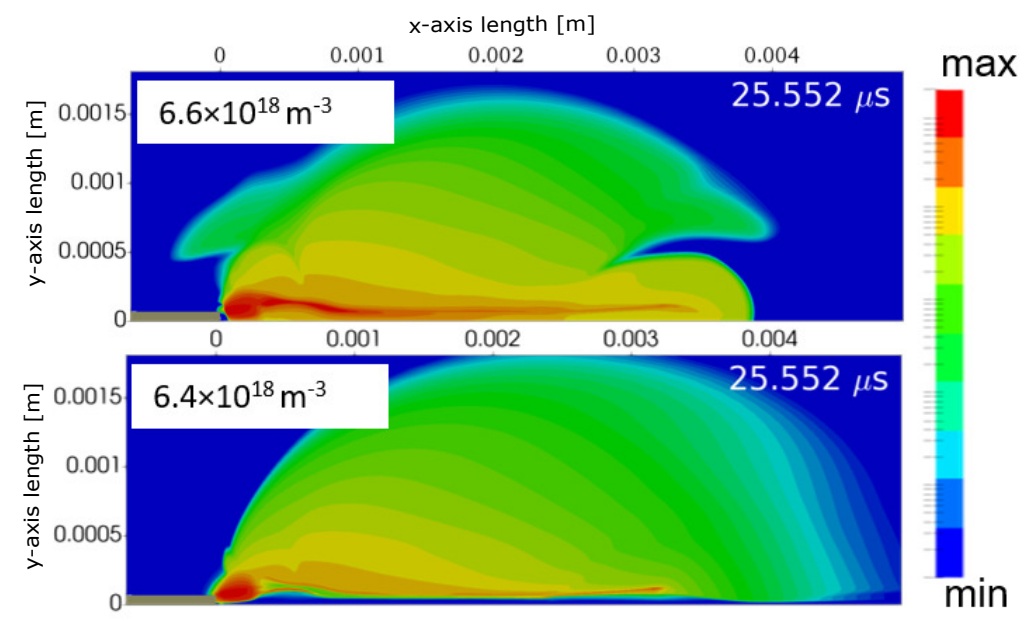

Figure 10. Positive (top) and negative (bottom) ion density distribution $\left[\mathrm{m}^{-3}\right.$, logscale] at the streamer relaxation regime in the positive phase (scaled to minimum of $10^{15} \mathrm{~m}^{-3}$ for visualization purposes - max values are indicated on the top-left corner of each time-snapshot). Both ion population is high inside an elongated zone due to the streamer relaxation.

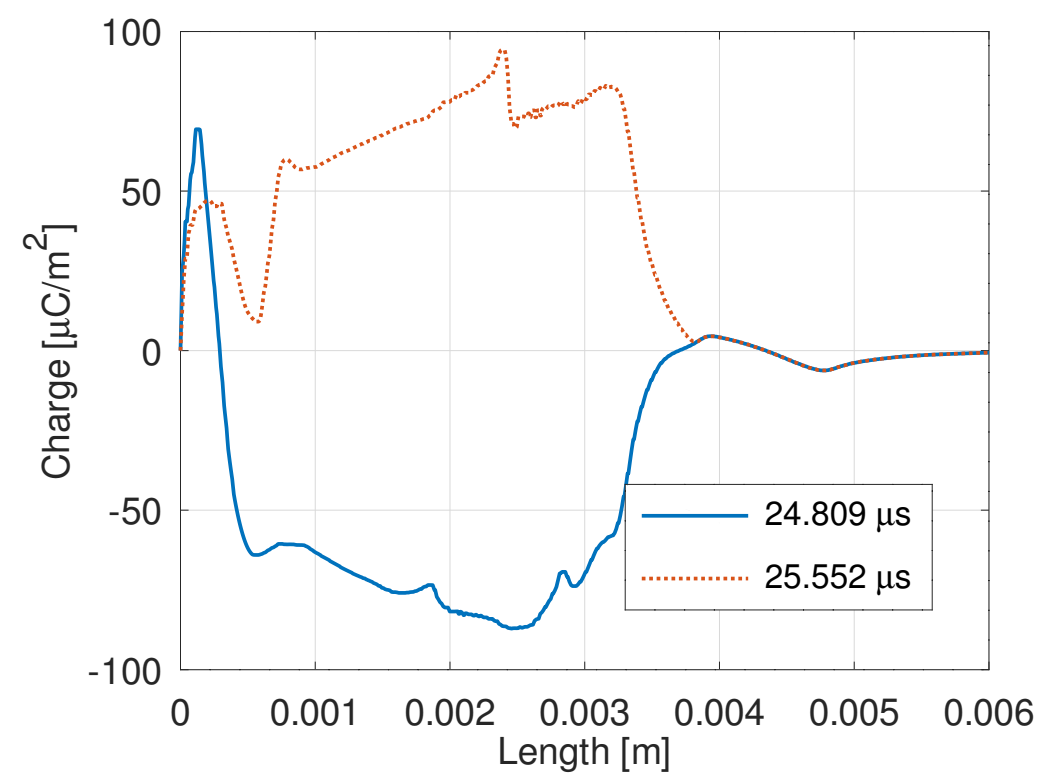

Figure 11. Surface charge for times $t=24.809 \mu \mathrm{s}$ and $t=25.552 \mu \mathrm{s}$ (right before and after the streamer discharge - see Fig. 6). The streamer discharge results to the positive charging of the dielectric surface.

surface charging effects as well as negative ion accumulation similar (but not same) as typical Trichel pulses. Here we choose to investigate in detail the first microdischarge as shown in the zoomed embedded figure in Fig. 12.

Fig. 13 shows the electron temperature for different time-instants during the microdischarge formation and relaxation while fig. 14 shows the electron density distribution at same time-instants. Moreover, Fig. 15 shows the potential distribution 


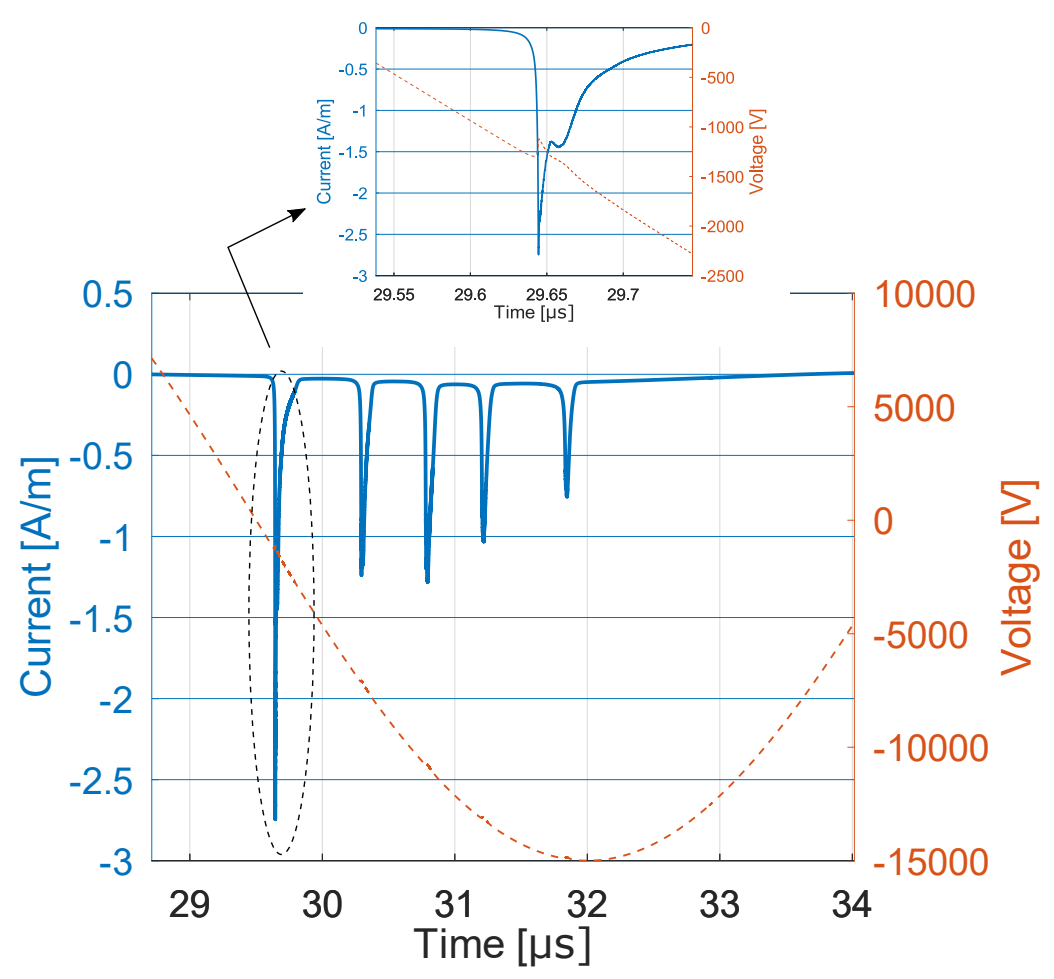

Figure 12. Current vs time in the negative phase. The zoomed embedded figure on the top shows the current vs time during the first microdischarge.

along with EHD force vectors.

When the negative phase begins $(t=29.557 \mu \mathrm{s})$, electrons created in the electrode ionization zone (near the active electrode) are quickly repelled from the active electrode and drift towards the dielectric layer. On the contrary, positive ions (still present in the volume from the positive phase as seen in Fig. $17-t=29.557 \mu \mathrm{s}$ ) follow the opposite path leading to a strong charge separation near the corner of the active electrode (negative ions contribute too to the negative volume charge at this region). Note that positive ions are completely absent near the dielectric surface as they are being repelled by the positive surface potential remaining from the previous phase. The strong electric field due to this space charge separation initiates the micro-discharge essential a positive going micro-streamer which quickly attaches to the electrode forming a cathode layer $(t=29.647 \mu s-t=29.668 \mu s)$. The thickness of the cathode layer is approx. $20 \mu \mathrm{m}$ and it surrounds the electrode corner. The electric field is located inside the cathode layer where strong ionization occurs. The cathode burning lasts some 10 s of ns and the born electrons quickly drift towards the dielectric surface charging it negatively close to the active electrode (Fig. 14, $t=29.654 \mu \mathrm{s}$ ). Fig. 16 shows the surface charge on the dielectric surface right before and right after a microdischarge formation (the cathode layer region is visible as the positively charged thin region near the cathode). The potential difference between this negatively charged portion 


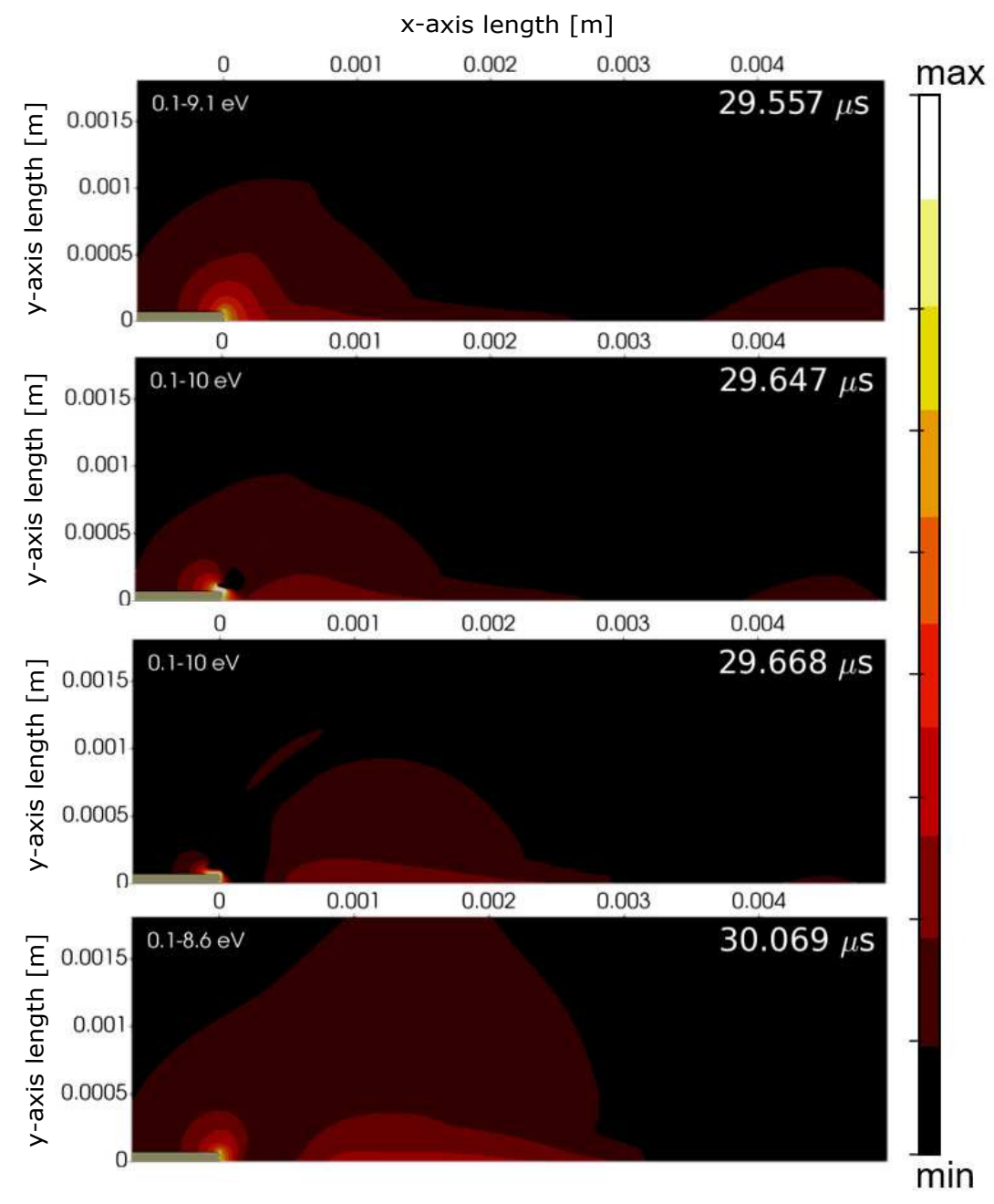

Figure 13. Electron Temperature $[\mathrm{eV}]$ during the first negative microdischarge pulse (min and max values are indicated on the top-left corner of each time-snapshot; the maximum temperature for subfigure 2 and 3 have been scaled to $10 \mathrm{eV}$ for visualization purposes). The microdischarge forms near the exposed electrode attracted by the latter and forming a high energy cathode sheath until it relaxes allowing for the development of the next microdischarge.

and the remaining positively charged layer leads to an increase of the electric field (and electron temperature, see Fig. $13 t=29.647 \mu s-t=29.668 \mu s$ ) very near the dielectric surface. Positive ions created in this region slowly drift towards the cathode and the positive ion cloud expands on the dielectric as the ionizing electric field region moves downstream. At the same time, negative ions (see Fig. 18) also contribute to the dielectric charging near the cathode. As the electrons now need to drift further away on the dielectric due to the negative surface charge (Fig. 14, $t=29.669 \mu s$ ), they lose their energy and slowly balance (or even reverse the charge of) a portion of the previously positive surface charge portion. The near-cathode negative charged portion is continuously fed with negative charges (mainly electrons) leading to the termination of the microdischarge as losses dominate over ionization at the cathode region due to the 


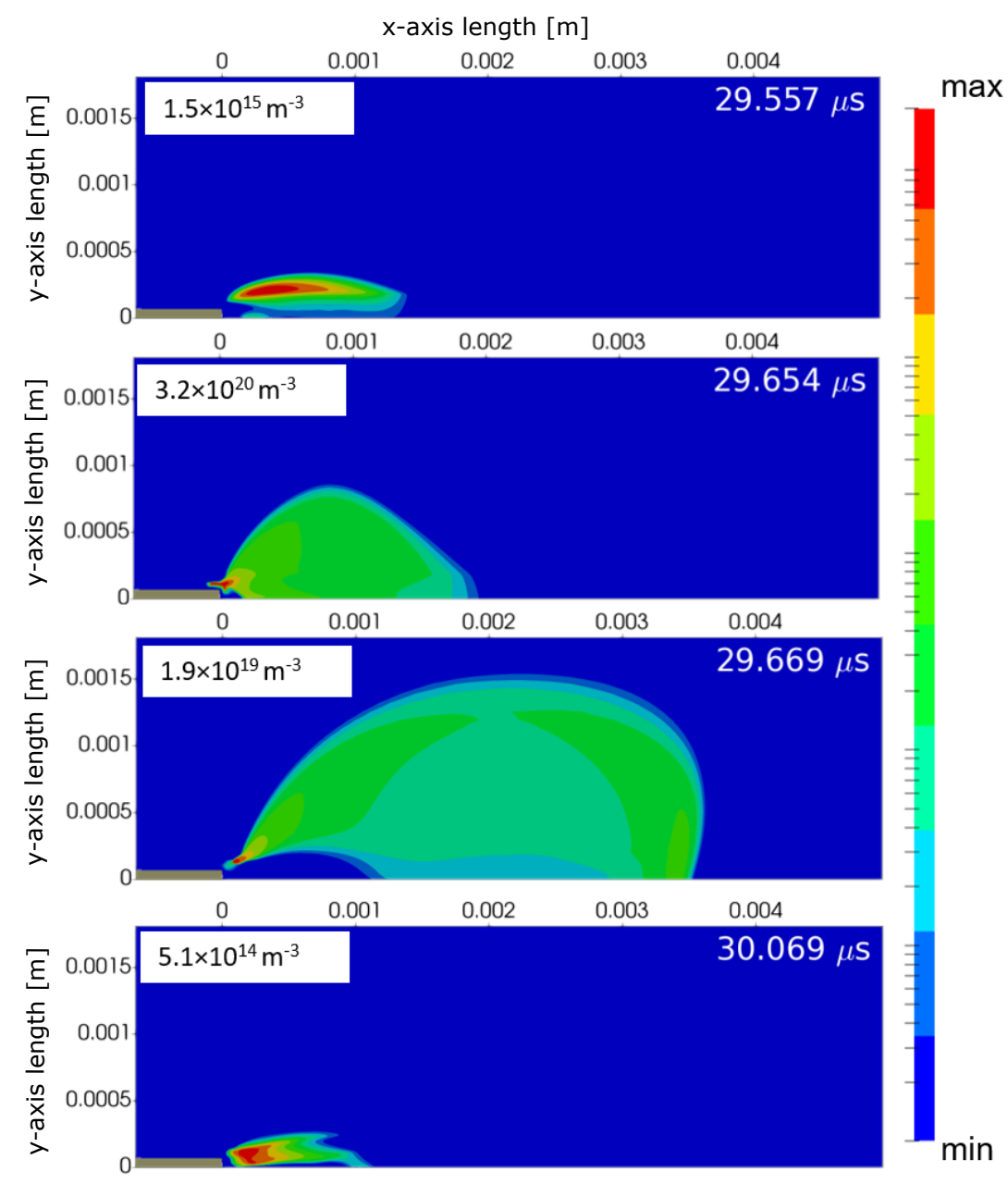

Figure 14. Electron density distribution $\left[\mathrm{m}^{-3}\right.$, log-scale] for different times during the first negative microdischarge pulse (scaled to minimum of $10^{14} \mathrm{~m}^{-3}$ for visualization purposes - max values are indicated on the top-left corner of each time-snapshot).

locally reduced electric field. The cathode layer detaches from the cathode and a thin plasma layer is formed attached to the dielectric surface similar to a weak surface anodedirected (negative) streamer. The initiation of this plasma surface discharge is due to the potential difference between the near cathode negatively charged region and the downstream positively charged portion of the dielectric. The plasma layer propagates to a distance of $1 \mathrm{~mm}$ on the dielectric surface and it's thickness is less than $70 \mu \mathrm{m}$ - it essentially propagates inside the streamer induced region linking the negative and positive phases strongly. Electrons are quickly lost mainly towards the positive portion of the dielectric (which persists from the positive-streamer phase). In subsequent times, positive ions are repelled outward from the near surface region leaving a layer of negative ions to populate this region $(t=30.069 \mu \mathrm{s})$. During the microdischarge burn (Fig. 15 - $t=29.654$ and $t=29.769 \mu \mathrm{s})$, the main part of the EHD force is located inside the cathode layer and it's strongly negative. The detachment of the cathode layer leads to the fast expulsion of negative ions from the cathode's corner leading to a short-lived 


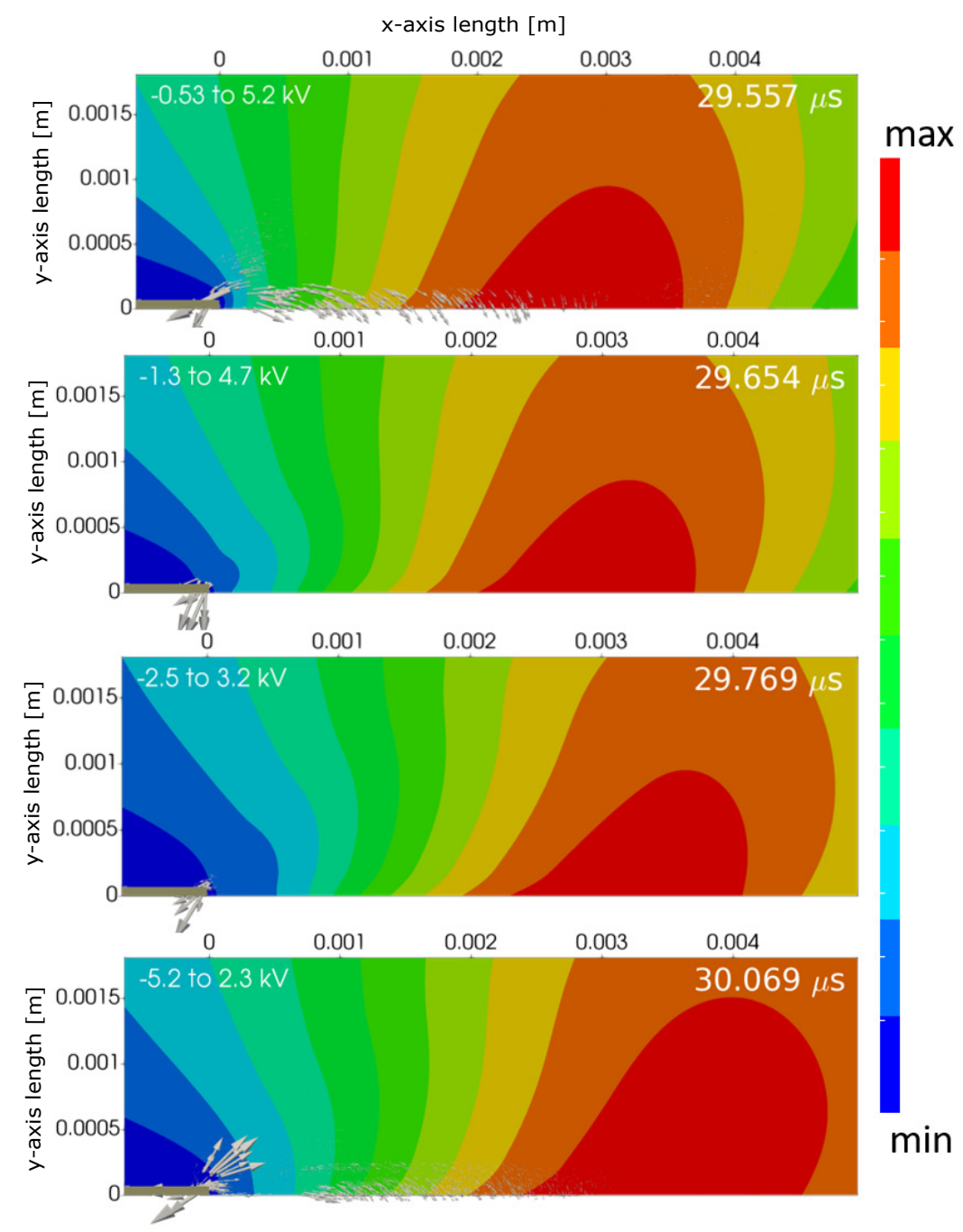

Figure 15. Potential distribution $[\mathrm{V}]$ (min and max values are indicated on the topleft corner of each time-snapshot) for different times in the negative phase during the first microdischarge. The EHD force vector field is also superimposed (vectors are scaled by their magnitude: Min value $=0 \mathrm{~N} / \mathrm{m}^{3}$, Max values are $1.2 \times 10^{6}, 1 \times 10^{9}$, $6.4 \times 10^{7}, 9.6 \times 10^{6} \mathrm{~N} / \mathrm{m}^{3}$ from top to bottom). The positive potential zone from the positive phase still persists during the first microdischarge.

positive EHD force at this region (Fig. $15-t=30.069 \mu \mathrm{s}$ ) while in parallel and during the subsequent relaxation phase, negative ions contribute to a positive EHD forcing very close to the dielectric surface. See Ref. [28] for more details on EHD distribution.

As the applied potential further decreases this process repeats for each subsequent microdischarge. A new microdischarge burn phase forms while electrons and negative ions charge the dielectric layer further downstream of the previous negative charged portion. The collapse of the cathode layer as explained above is followed by the initiation of the surface plasma layer. At each microdischarge pulse repetition, this plasma layer initiates further downstream as the negative charged region near the cathode is constantly expanding. This leads to a constantly expanding layer of charged 


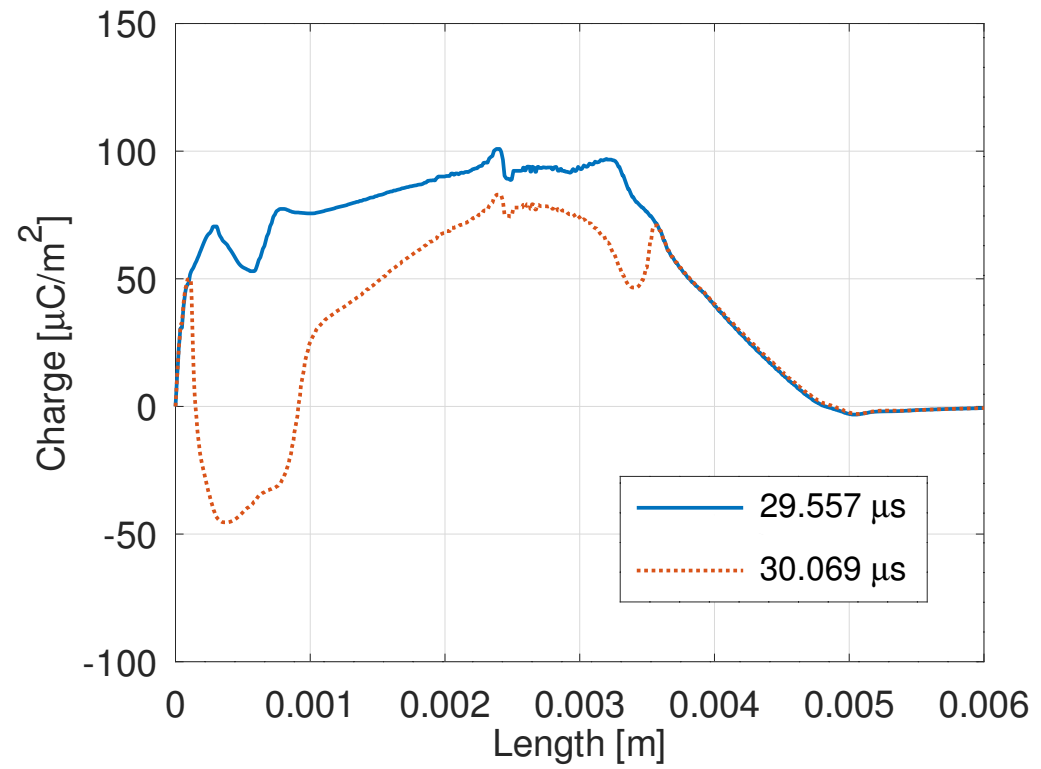

Figure 16. Surface charge for times $t=29.557 \mu \mathrm{s}$ and $t=30.069 \mu \mathrm{s}$ (right before and after the first microdischarge pulse - see Fig. 12). The dielectric portion of approximately $1 \mathrm{~mm}$ near the exposed electrode holds a negative charge now.

particles near the dielectric surface. Fig. 19 shows the positive and negative ion density distribution after the last microdischarge pulse. The ion layer has expanded at a distance of approx. $3.5 \mathrm{~mm}$ which is clearly linked to the positive streamer phase elongation and consequent positive dielectric charging. The negative ion layer expands due to the strong potential difference between negative and positive charged surface layers which can only be a result of the strong interaction between the two subcycles of the AC-DBD actuator. We also note (as it is evident from Fig. 19) that the positive ion cloud is mainly detached from the dielectric surface while the contrary pertains for the negative cloud. This implies that during the negative phase (especially the relaxation phase between each microdischarge) the EHD force is mainly located in the layer attached to the dielectric surface (see Ref. [28] for more details).

\section{Discussion}

The results presented here show a good qualitative agreement with previous studies while revealing important aspects of the surface AC-DBD two-phase operation. Nevertheless, some strong assumptions made (necessary in plasma fluid models) need further exploration. Comments on the assumptions made and limitations of the numerical approach used in this work are presented below.

- The AC frequency simulated in this work is higher than typical frequencies used in experiments $(1-25 \mathrm{kHz})$. A lower frequency (under same applied voltage) leads to several streamers and more microdischarges in both subcycles as has been shown experimentally. Although this fact will modify the overall discharge dynamics 


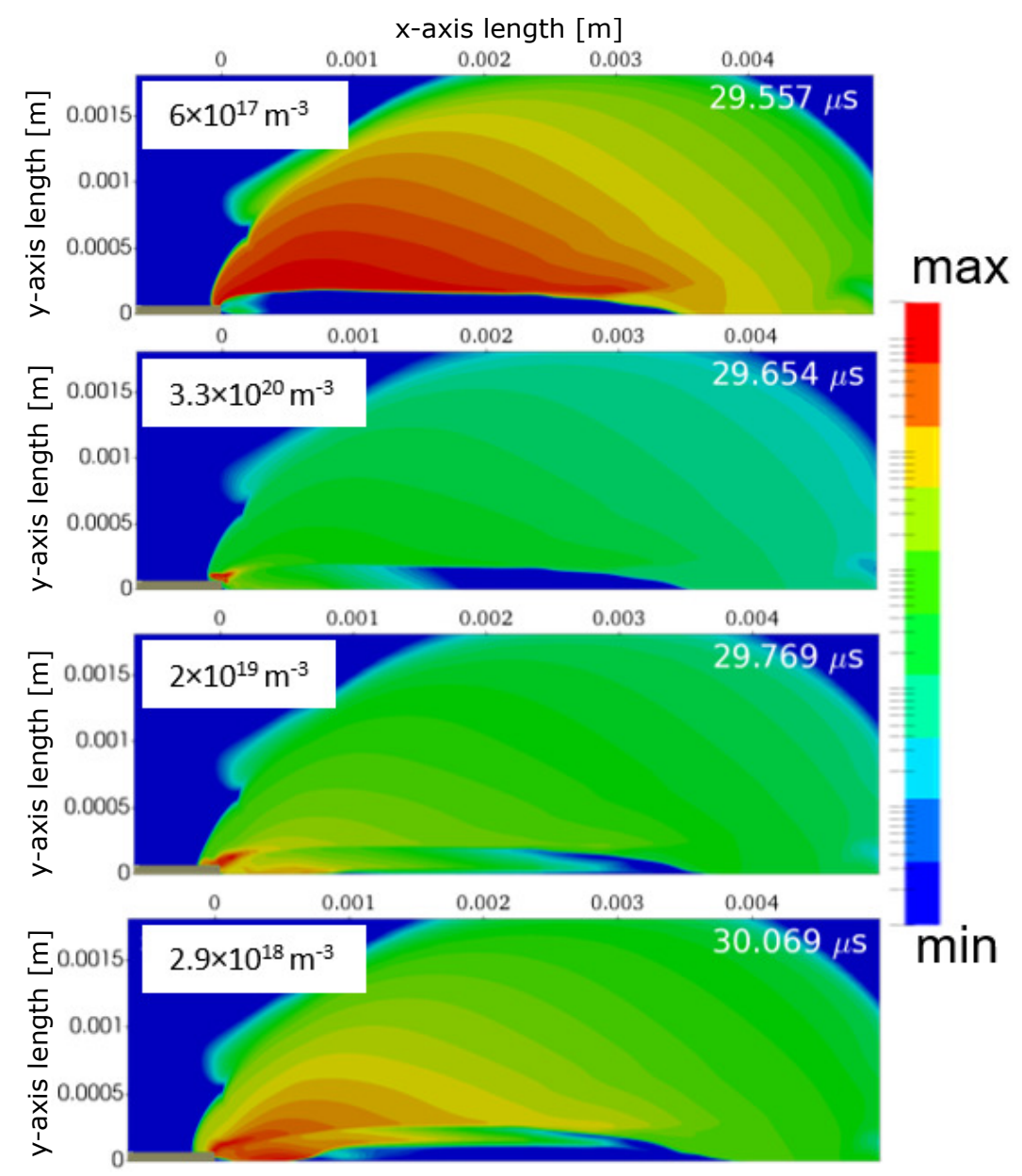

Figure 17. Positive ion density distribution $\left[\mathrm{m}^{-3}\right.$, log-scale $]$ during one negative microdischarge pulse (scaled to minimum of $10^{15} \mathrm{~m}^{-3}$ for visualization purposes - max values are indicated on the top-left corner of each time-snapshot). See text for details.

(length of ion cloud propagation, streamer propagation, memory effects etc), the results presented here should apply qualitatively even in lower frequencies. Each streamer will go through a preburn (glow/corona like regime), burn and afterburn (relaxation) phase. Similar for each microdischarge. We have already confirmed this claim at frequencies of $50 \mathrm{kHz}$. The repetition rate and its detailed influence on relevant dynamics is left for a future study (simulations are on-going for frequencies of $10 \mathrm{kHz}$ and lower).

- AC-DBDs are known to be non-thermal. Joule heating occurs locally near the exposed electrode with very mild temperature rise. In frequencies such as $100 \mathrm{kHz}$, heating could be important as energy relaxation may not have time to occur. Still, even for such high frequencies, we are still far from the nanosecond regime [38], known for its thermal nature due to ultra-fast gas heating.

- Photo-ionization has not been used in the current work. Even though its influence on streamer initiation and propagation (as well as sustainability of corona regime) is expected to be important, its effects are expected to be limited to faster initiation of 


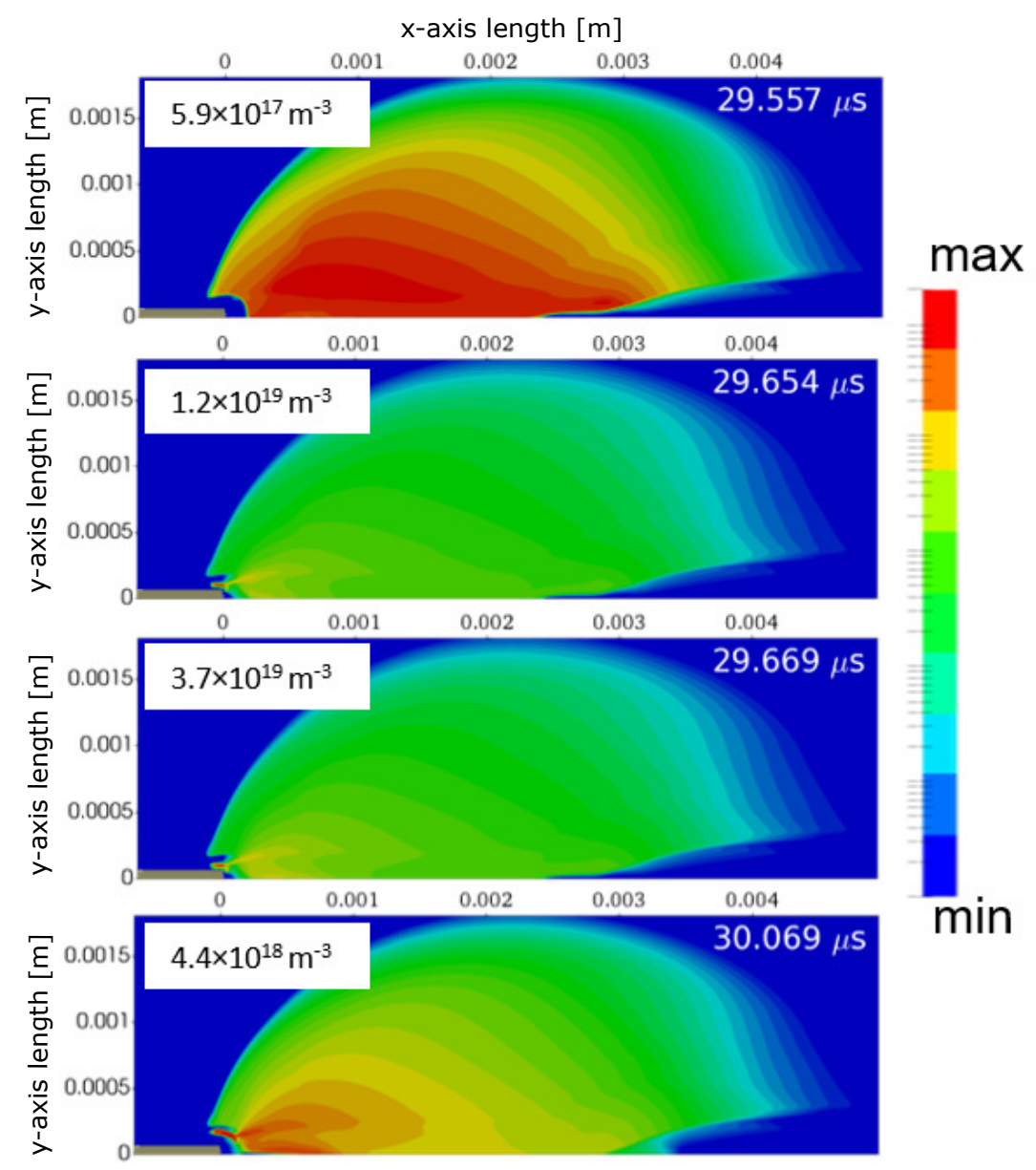

Figure 18. Negative ion density distribution $\left[\mathrm{m}^{-3}, \log\right.$-scale $]$ during one negative microdischarge pulse (scaled to minimum of $10^{15} \mathrm{~m}^{-3}$ for visualization purposes - max values are indicated on the top-left corner of each time-snapshot). See text for details.

the discharge and longer elongation of the streamer [39]. Photoionization provides means of free electrons which enhance the propagation of a positive streamer discharge especially under low applied voltages. In the conditions studied in this paper, the applied voltage is high enough to initiate the streamer discharge and the seed electrons are provided by the background ionization level. Ref. [40] also confirms the reduced influence of the photoionization in the overall physics of surface DBD actuators. Several numerical studies have used similar approaches (Ref. [14, 13]. We have also confirmed this fact via streamer simulations including photo-ionization: the surface streamer initiation is slightly accelerated while its propagation is elongated without further influence on the AC discharge properties. Thus, qualitatively the claims made in this work remain unchanged and valid. Mimicking its effect with a background ionization level, can be considered a safe assumption for the points made while significantly reducing the CPU time. Quantitatively, the longer elongation of the streamer discharge (2-3 times faster propagation according to Ref. [39] with an increase of the streamer thickness) should 


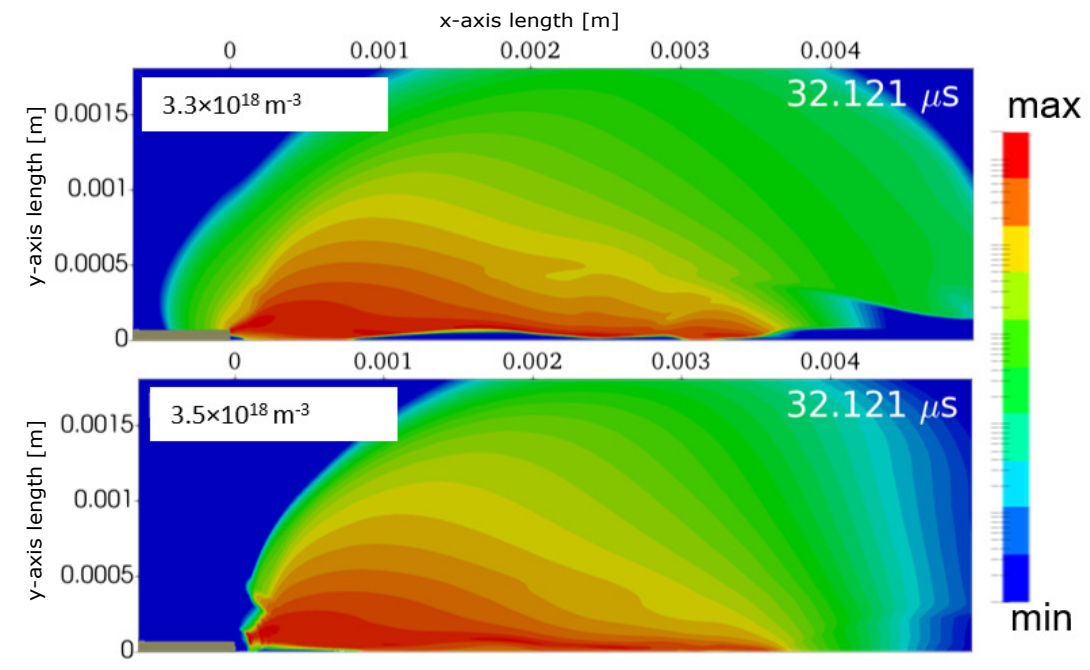

Figure 19. Positive (top) and negative (bottom) ion density distribution $\left[\mathrm{m}^{-3}, \log\right.$ scale] just after the last microdischarge pulse (scaled to minimum of $10^{15} \mathrm{~m}^{-3}$ for visualization purposes - max values are indicated on the top-left corner of each timesnapshot).

lead to higher currents and a global elongation of the discharge zone (including the surface charging region). Considering the focus of this paper on physical insights and not an one-to-one comparison with experiments along with the ambiguity of photoionization models under high electric fields, we leave a more detailed study including photoionization for a future work.

- The plasma chemistry used in this work is quite simplified. The complex reactions and rich chemistry in atmospheric pressure air requires such simplifications in order to obtain models that are accurate but also computationally efficient. Nevertheless, such simplified chemistry has been proven to be adequate for reproducing the main attributes of this actuator $[13,16]$. Reactions such as super-elastic collisions, ion-ion exchange etc could have implications (at various ranges of reduced electric fields) but should not greatly influence the discharge phases. Moreover, the saturation of swarm parameters at high voltages cannot be correctly calculated by two-term solvers such as BOLSIG+. We have performed several studies on the validity of the main reaction parameters (especially ionization) at high voltages and concluded that a limitation of the ionization coefficient at reduced fields in the order of 1200 $\mathrm{Td}$ is necessary to stabilize the simulations especially using the LFA. Under the LMEA though, the discharge stabilizes easier even at regions of very high electric field (near the exposed electrode, cathode sheath or streamer head) due to the electron energy balance and diffusion/wall losses. A more detailed study with a more complex chemistry is left for future studies.

- The computational mesh used in this study has been adapted to capture all features of the discharge especially the streamer propagation and microdischarge formation (along with the cathode sheath). These features dictate a cell size in the order of 1 
$\mu \mathrm{m}$ which renders the simulations quite expensive. A detailed mesh independence study with the use of adaptive mesh refinement techniques is also left for a future study.

- The nature of the surface DBD is three-dimensional (3D). Discharges (both streamers and microdischarges) are initiated on spots of the exposed electrode with intervals on the third-dimension which are linked to chaotic procedures and impurities of the electrodes. The two-dimensional (2D) approach used here is thus limited to model uniform discharges in the third dimension, neglecting any interactions between neighboring discharges. Such interactions may lead to streamer branching as observed in experiments while the electric field interaction between neighboring discharges might also influence the development of each discharge. Three-dimensional simulations in Ref. [20] using artificial disturbances to initiate the spanwise non-uniform discharges have revealed that the average spatiotemporal evolution of the body force (which strongly relates to charge and electric field distribution) is well reproduced by the $2 \mathrm{D}$ models despite the nonuniformity observed. Moreover, experiments have demonstrated that neighboring discharges are typically located several mm (see Ref. [9] for example) apart possibly underlying a self-organizing structure of the spanwise discharge which eliminates high interference. Therefore, the 2D approximation used in this study can be considered as a relatively good approximation for the evolution of both the streamer and the microdischarge regimes.

\section{Concluding Remarks}

The detailed numerical study of the surface AC-DBD actuator demonstrates the importance of both positive and negative cycles on the overall discharge evolution. The streamer discharge propagates detached from the dielectric surface (as well as the active electrode) and plays the role of a virtual anode which enhances the electric field at the streamer's head after the termination of its propagation. The streamer regime and especially its relaxation phase leads to the deposition of positive charges on the dielectric surface which occupy a region linked to the streamer elongation. During the negative phase, microdischarges are formed followed by the formation of a plasma layer attached to the dielectric surface. The cathode layer during the microdischarge burn phase feeds both the volumetric and surface charge population while the plasma layer expands further and further during each microdischarge linked to the potential difference between negatively and positively charged surface regions. The latter observation leads to the conclusion that both phases are strongly connected and the plasma expansion to several millimetres can be only explained by taking into account this interaction in a detailed study as this work. This plasma expansion implies important consequences in the EHD force spatial distribution and subsequent ion wind flow including the localization of their maximum values to several millimetres from the exposed electrode as observed in experiments [32]. These consequences are highlighted in Ref. [28] focusing on the EHD 
force spatiotemporal profiles. Our findings can serve towards the optimization of surface AC-DBD actuators into efficient flow control methods, contribute to the understanding of such devices and underlying physics and open new paths of research in the field of plasma-fluid interactions.

\section{Acknowledgments}

This work has received funding under the E.U. MSCA-RISE - Marie Skłodowska-Curie Research and Innovation Staff Exchange (RISE) scheme as part of the project 'Control of Turbulent Friction Force' (CTFF) - Grant agreement ID: 777717.

\section{References}

[1] Kirill V Khodataev. Microwave discharges and possible applications in aerospace technologies. Journal of Propulsion and Power, 24(5):962-972, 2008.

[2] Yu Kolesnichenko, V Brovkin, D Khmara, V Lashkov, I Mashek, and M Ryvkin. Microwave discharge parameters in supersonic flow. In 40th AIAA Aerospace Sciences Meeting 83 Exhibit, Reno, NV, pages 14-17, 2002.

[3] Konstantinos Kourtzanidis. Modélisation numérique d'actionneurs plasma pour le contrôle d'écoulement. PhD thesis, Toulouse, ISAE, 2014.

[4] KR Grossman, BZ Cybyk, DM VanWie, et al. Sparkjet actuators for flow control. AIAA paper, 57:2003, 2003.

[5] G Sary, G Dufour, F Rogier, and K Kourtzanidis. Modeling and parametric study of a plasma synthetic jet for flow control. AIAA journal, 52(8):1591-1603, 2014.

[6] Eric Moreau. Airflow control by non-thermal plasma actuators. Journal of Physics D: Applied Physics, 40(3):605, 2007.

[7] Thomas C Corke, C Lon Enloe, and Stephen P Wilkinson. Dielectric barrier discharge plasma actuators for flow control. Annual review of fluid mechanics, 42:505-529, 2010.

[8] J Reece Roth, Daniel M Sherman, and Stephen P Wilkinson. Electrohydrodynamic flow control with a glow-discharge surface plasma. AIAA journal, 38(7):1166-1172, 2000.

[9] Nicolas Benard and Eric Moreau. Electrical and mechanical characteristics of surface ac dielectric barrier discharge plasma actuators applied to airflow control. Experiments in Fluids, 55(11):1846, 2014.

[10] A Debien, N Benard, L David, and E Moreau. Unsteady aspect of the electrohydrodynamic force produced by surface dielectric barrier discharge actuators. Applied Physics Letters, 100(1):013901, 2012.

[11] Yildirim Suzen, George Huang, Jamey Jacob, and David Ashpis. Numerical simulations of plasma based flow control applications. In 35th AIAA Fluid Dynamics Conference and Exhibit, page 4633, 2005.

[12] JP Boeuf and LC Pitchford. Electrohydrodynamic force and aerodynamic flow acceleration in surface dielectric barrier discharge. Journal of Applied Physics, 97(10):103307, 2005.

[13] JP Boeuf, Y Lagmich, Th Unfer, Th Callegari, and LC Pitchford. Electrohydrodynamic force in dielectric barrier discharge plasma actuators. Journal of Physics D: Applied Physics, 40(3):652, 2007.

[14] Alexandre V Likhanskii, Mikhail N Shneider, Sergey O Macheret, and Richard B Miles. Modeling of dielectric barrier discharge plasma actuator in air. Journal of Applied Physics, 103(5):053305, 2008.

[15] PC Dörr and MJ Kloker. Numerical investigation of plasma-actuator force-term estimations from flow experiments. Journal of Physics D: Applied Physics, 48(39):395203, 2015. 
[16] M Abdollahzadeh, JC Pascoa, and PJ Oliveira. Implementation of the classical plasma-fluid model for simulation of dielectric barrier discharge (dbd) actuators in openfoam. Computers 83 Fluids, 128:77-90, 2016.

[17] VR Soloviev and VM Krivtsov. Surface barrier discharge modelling for aerodynamic applications. Journal of Physics D: Applied Physics, 42(12):125208, 2009.

[18] VR Soloviev. Analytical model of a surface barrier discharge development. Plasma Physics Reports, 45(3):264-276, 2019.

[19] Hiroyuki Nishida and Takashi Abe. Numerical analysis of plasma evolution on dielectric barrier discharge plasma actuator. Journal of applied physics, 110(1):013302, 2011.

[20] Hiroyuki Nishida, Taku Nonomura, and Takashi Abe. Three-dimensional simulations of discharge plasma evolution on a dielectric barrier discharge plasma actuator. Journal of Applied Physics, 115(13):133301, 2014.

[21] G Dufour and F Rogier. Numerical modeling of dielectric barrier discharge based plasma actuators for flow control: the copaier/cedre example. AerospaceLab, 2015.

[22] Anne Bourdon, VP Pasko, NY Liu, Sébastien Célestin, Pierre Ségur, and Emmanuel Marode. Efficient models for photoionization produced by non-thermal gas discharges in air based on radiative transfer and the helmholtz equations. Plasma Sources Science and Technology, 16(3):656, 2007.

[23] Graeme G Lister. Low-pressure gas discharge modelling. Journal of Physics D: Applied Physics, 25(12):1649, 1992.

[24] GJM Hagelaar, FJ De Hoog, and GMW Kroesen. Boundary conditions in fluid models of gas discharges. Physical Review E, 62(1):1452, 2000.

[25] HW Ellis, RY Pai, EW McDaniel, EA Mason, and LA Viehland. Transport properties of gaseous ions over a wide energy range. Atomic data and nuclear data tables, 17(3):177-210, 1976.

[26] Vasilios Alexiades, Geneviéve Amiez, and Pierre-Alain Gremaud. Super-time-stepping acceleration of explicit schemes for parabolic problems. Communications in numerical methods in engineering, 12(1):31-42, 1996.

[27] GJM Hagelaar and GMW Kroesen. Speeding up fluid models for gas discharges by implicit treatment of the electron energy source term. Journal of Computational Physics, 159(1):1-12, 2000 .

[28] Konstantinos Kourtzanidis, Guillaume Dufour, and François Rogier. Explaining the electrohydrodynamic force and ionic wind spatiotemporal distribution in surface ac dielectric barrier discharge actuators. arXiv preprint arXiv:2007.01614, 2020.

[29] Peter LG Ventzek, Robert J Hoekstra, and Mark J Kushner. Two-dimensional modeling of high plasma density inductively coupled sources for materials processing. Journal of Vacuum Science 83 Technology B: Microelectronics and Nanometer Structures Processing, Measurement, and Phenomena, 12(1):461-477, 1994.

[30] Andrea Villa, Luca Barbieri, Marco Gondola, and Roberto Malgesini. An asymptotic preserving scheme for the streamer simulation. Journal of Computational Physics, 242:86-102, 2013.

[31] Michael S Barnes, Tina J Cotler, and Michael E Elta. Large-signal time-domain modeling of low-pressure rf glow discharges. Journal of applied physics, 61(1):81-89, 1987.

[32] N Benard, P Note, M Caron, and E Moreau. Highly time-resolved investigation of the electric wind caused by surface dbd at various ac frequencies. Journal of Electrostatics, 88:41-48, 2017.

[33] Christophe Geuzaine and Jean-François Remacle. Gmsh: A 3-d finite element mesh generator with built-in pre-and post-processing facilities. International journal for numerical methods in engineering, 79(11):1309-1331, 2009.

[34] GJM Hagelaar and LC Pitchford. Solving the boltzmann equation to obtain electron transport coefficients and rate coefficients for fluid models. Plasma Sources Science and Technology, 14(4):722, 2005.

[35] Bolsig+ version 12/2017 accessed online.

[36] AV Phelps. Lxcat database, 2013. 
[37] Sergey Pancheshnyi, M Nudnova, and A Starikovskii. Development of a cathode-directed streamer discharge in air at different pressures: experiment and comparison with direct numerical simulation. Physical Review E, 71(1):016407, 2005.

[38] Konstantinos Kourtzanidis and Laxminaryan L Raja. Three-electrode sliding nanosecond dielectric barrier discharge actuator: modeling and physics. AIAA Journal, pages 1393-1404, 2017.

[39] Alexandre Likhanskii, Vladimir Semak, Dmitry Opaits, Mikhail Shneider, Richard Miles, and Sergey Macheret. The role of the photoionization in the numerical modeling of the dbd plasma actuator. In 47th AIAA Aerospace Sciences Meeting including The New Horizons Forum and Aerospace Exposition, page 841, 2009.

[40] Jean-Charles Matéo-Vélez. Modélisation et simulation numérique de la génération de plasma dans les décharges couronnes et de son interaction avec l'aérodynamique. PhD thesis, Toulouse, ENSAE, 2006. 